\title{
Dark Matter Searches Using NaI(Tl) at the Canfranc Underground Laboratory: Past, Present and Future
}

\author{
Julio Amaré 1,2, Susana Cebrián 1,2 ${ }^{\mathbb{D}}$, David Cintas ${ }^{1,2}$, Iván Coarasa ${ }^{1,2} \mathbb{D}$, Clara Cuesta ${ }^{3}$, Eduardo García ${ }^{1,2}$ (D) \\ María Martínez 1,2,4 D, Ángel Morales ${ }^{\dagger}$, Julio Morales ${ }^{\dagger}$, Miguel Ángel Oliván ${ }^{5}$, Ysrael Ortigoza 1,2, \\ Alfonso Ortiz de Solórzano ${ }^{1,2}$, Tamara Pardo ${ }^{1,2}$, Carlos Pobes ${ }^{6}$ (D), Jorge Puimedón ${ }^{1,2}$, Ana Salinas ${ }^{1,2}$, \\ María Luisa Sarsa ${ }^{1,2, * \mathbb{D}}$, José Ángel Villar ${ }^{\dagger}$ and Patricia Villar ${ }^{1,2}$
}

check for

updates

Citation: Amaré, J.; Cebrián, S.;

Cintas, D.; Coarasa, I.; Cuesta, C.;

García, E.; Martínez, M.; Morales, Á.;

Morales, J.; Oliván, M.Á.; et al. Dark

Matter Searches Using $\mathrm{NaI}(\mathrm{Tl})$ at the

Canfranc Underground Laboratory:

Past, Present and Future. Universe

2022, 8, 75. https://doi.org/10.3390/

universe 8020075

Academic Editor: Sergey Burdin

Received: 17 December 2021

Accepted: 18 January 2022

Published: 27 January 2022

Publisher's Note: MDPI stays neutral with regard to jurisdictional claims in published maps and institutional affiliations.

Copyright: (C) 2022 by the authors. Licensee MDPI, Basel, Switzerland. This article is an open access article distributed under the terms and conditions of the Creative Commons Attribution (CC BY) license (https:// creativecommons.org/licenses/by/ $4.0 /)$.
1 Centro de Astropartículas y Física de Altas Energías (CAPA), Universidad de Zaragoza, Pedro Cerbuna 12, 50009 Zaragoza, Spain; amare@unizar.es (J.A.); scebrian@unizar.es (S.C.); cintas@unizar.es (D.C.); icoarasa@unizar.es (I.C.); edgarcia@unizar.es (E.G.); mariam@unizar.es (M.M.); ortigoza@unizar.es (Y.O.); alfortiz@unizar.es (A.O.d.S.); tpardo@unizar.es (T.P.); puimedon@unizar.es (J.P.); salinas@unizar.es (A.S.); pvillar@unizar.es (P.V.)

2 Laboratorio Subterráneo de Canfranc, Paseo de los Ayerbe, 22880 Canfranc Estación, Spain

3 Centro de Investigaciones Energéticas y Medioambientales (CIEMAT), Avda. Complutense 40, 28040 Madrid, Spain; clara.cuesta@ciemat.es

4 Fundación ARAID, Avenida de Ranillas 1D, 50018 Zaragoza, Spain

5 Fundación CIRCE, Avenida de Ranillas 3D, 50018 Zaragoza, Spain; maolivan@unizar.es

6 Instituto de Nanociencia y Ciencia de Materiales de Aragón (INMA), CSIC-Universidad de Zaragoza, Pedro Cerbuna 12, 50009 Zaragoza, Spain; cpobes@unizar.es

* Correspondence: mlsarsa@unizar.es

$\dagger$ Deceased.

\begin{abstract}
Sodium Iodide Thallium doped $(\mathrm{NaI}(\mathrm{Tl}))$ scintillation detectors have been applied to the direct searches for dark matter since the 1980s and have produced one of the most challenging results in the field-the observation by the DAMA/LIBRA collaboration of an annual modulation in the detection rate for more than twenty cycles. This result is very difficult to reconcile with negative results derived from other experiments using a large variety of target materials and detection techniques. However, it has been neither confirmed nor refuted in a model independent way up to the present. Such a model independent test of the DAMA/LIBRA result is the goal of the ANAIS-112 experiment, presently in the data taking phase at the Canfranc Underground Laboratory in Spain. ANAIS-112 design and operation leans on the expertise acquired at the University of Zaragoza in direct searches for Dark Matter particles using different targets and techniques and in particular using $\mathrm{NaI}(\mathrm{Tl})$ scintillation detectors for about thirty years, which are reviewed in the first section of this manuscript. In addition to presenting the status and more recent results of the ANAIS-112 experiment, open research lines, continuing this effort, will be presented.
\end{abstract}

Keywords: dark matter; $\mathrm{NaI}(\mathrm{Tl})$ scintillators; annual modulation; DAMA/LIBRA puzzle; Laboratorio Subterráneo de Canfranc

\section{Introduction}

For almost a century, evidence coming from the Universe at different scales points to the existence of an unknown matter component in galaxies, clusters of galaxies and cosmological scales. The $\Lambda$ CDM cosmological model satisfactorily explains the observations at the cost of introducing $84 \%$ of the matter in the form of Dark Matter (DM) and $68 \%$ of the energy in the form of Dark Energy (DE) [1,2]. Proposed alternative theories based on modified gravity or dynamics are not capable, at this moment, of reproducing observations without DM or DE.

Understanding the nature of the DM has been shown to be a challenge requiring complementary approaches from the different points of view of cosmology, astronomy, 
astrophysics, and nuclear and particle physics (theoretical and experimental). Despite the large efforts invested in the last decades following different strategies (searches at accelerators [3], indirect [4] and direct searches [5-7]), no clues on the nature of DM are yet at hand.

In the context of direct DM searches, the goal is to measure the small energy deposited in the target material by the interaction of the DM particles filling the Milky Ways's halo at the Solar System position. The expected signal is strongly dependent on the particle DM model (mass, kind and magnitude of the coupling to quarks and leptons), and on the galactic halo model (density of DM and velocity distribution function, both at the Solar System position). Those models' parameters are either unknown or affected by large uncertainties. In addition, those parameters interfere with the target nuclei properties in such a way that comparison between results from experiments using different absorber materials are model-dependent. The low interaction rates of these DM particles imply that backgrounds have to be minimized by operating the experiment underground, by using radiopure materials in the detector building, by shielding conveniently (passively and/or actively) against external backgrounds, and profiting from any background rejection procedure at hand. After more than three decades of DM direct searches, most of the experimental results are compatible with the estimated backgrounds. At present, there are two relevant results requiring further understanding: the XENON-1T experiment observes an excess of events that can be attributed either to tritium contamination or exotic particles interactions (axions, neutrino magnetic moment, warm dark matter, etc.) [8,9]; and DAMA/LIBRA result in annual modulation that lasts for more than two decades and cannot be explained by any known background (see below).

For a positive identification of the DM in direct detection searches it is necessary to analyze distinctive features of a DM signal against backgrounds. This can be done by studying the annual modulation effect in the DM interaction rates [10] produced by the orbital motion of the Earth around the Sun along the year. The corresponding variation in the relative velocity of the DM particles with respect to the target nuclei induces a modulation with a one-year period and a maximum at about the 2nd of June in the DM interaction rate, for the standard halo model.

Sodium Iodide Thallium doped $(\mathrm{NaI}(\mathrm{Tl}))$ scintillation detectors are a convenient choice for detecting radiation in many applications. In particular, they have been applied to the direct searches for dark matter since the 1980s and have produced one of the most challenging results in the field - the observation by DAMA/LIBRA collaboration of an annual modulation in the detection rate for more than twenty years [11-16]. This result is very difficult to reconcile with the negative results derived from other experiments using a large variety of target materials and detection techniques [17-33]. However, it has neither been confirmed nor refuted in a model independent way up to the present. The DAMA/LIBRA signal is fully compatible with the annual modulation associated to DM particles distributed in our galaxy in a halo with standard properties, and all the systematic effects proposed to contribute to the signal have been discarded by the DAMA/LIBRA collaboration. It is important to solve this experimental puzzle, which could be the door into new physics, and in order to do so a model independent test needs to be faced. Although the DAMA/LIBRA result is incompatible with the rest of the experiments searching for $\mathrm{DM}$ in most of the analyzed scenarios, it should be stressed that this comparison is model dependent and the DM nature is still fully unknown.

A model-independent test of DAMA/LIBRA result requires an experiment using the same target material, a similar background and threshold energy to those of DAMA/LIBRA, large exposure (product of mass and measurement time) and stability, in order to analyze the annual modulation effect. This is the goal of the ANAIS-112 experiment, presently in the data taking phase at the Canfranc Underground Laboratory, in Spain. The ANAIS-112 design and operation leans on the expertise acquired at the University of Zaragoza using $\mathrm{NaI}(\mathrm{Tl})$ scintillation detectors for about thirty years, which is reviewed in this manuscript and has been collected in several PhD dissertations [34-41]. 
Other experiments all over the world share the ANAIS-112 goal. The COSINE-100 experiment is also in the data taking phase at the YangYang laboratory [42,43], in the Republic of Korea, with an effective mass of about $60 \mathrm{~kg}$, instead of the $112.5 \mathrm{~kg}$ of ANAIS-112, and the $250 \mathrm{~kg}$ of DAMA/LIBRA detector. SABRE [44], PICOLON [45] and COSINE-200 are still in $R \& D$ phase, aiming at improving the radiopurity of the $\mathrm{NaI}(\mathrm{Tl})$ crystals, but using similar technology. COSINUS is also in the R\&D phase, but using a completely different detection approach, based on the simultaneous measurement of the light and phonons produced following a particle interaction in the $\mathrm{NaI}(\mathrm{Tl})$ crystal, which allows identifying the particle by the different sharing between the two energy conversion channels [46]. Previously, DM-Ice17, operating at the South Pole, tried to address this goal, but its sensitivity was too low [47].

Thallium doped sodium iodide is a high-performing scintillating material: large size crystals can be grown (up to tens of kilograms) and the amount of light produced by unit of energy deposited is one of the highest among scintillating materials, at the level of 40,000 photons / MeV. The wavelength of the light produced matches perfectly with the peak sensitivity of bi-alkali PMTs, easing the fabrication of scintillation detectors. In the application to DM searches, the $100 \%$ non-zero spin of both component nuclei, ${ }^{127}$ I and ${ }^{23} \mathrm{Na}$, makes $\mathrm{NaI}$ a very interesting target for the case of spin-dependent interacting DM particle candidates, and the combination of heavy and light nuclei allows a good kinematical matching in a wide range of DM particle masses.

However, the radioactive background level achieved by $\mathrm{NaI}(\mathrm{Tl})$ detectors has practically not improved in the last twenty years. There are several reasons behind, on the one hand, raw powder purification and crystal growing technology of BICRON/Saint Gobain at the end of the last century was not replicated by other companies, and further efforts to go beyond that level date from the last decade. On the other hand, the use of PMTs for the light readout of $\mathrm{NaI}$ scintillation light allowed the rise of the technology, but it is at present a drawback to increase its sensitivity. PMTs are not radiopure enough and contribute strongly to the radioactive background budget of the detectors. Moreover, they produce spurious events interfering with the bulk scintillation light detection in a way which has shown to be very tough to keep under control, limiting the detection of small amounts of light and, as a consequence, further reduction of the achievable energy threshold. Because of that, other light detectors coupled to the $\mathrm{NaI}$ scintillating crystals are under study in order to improve the sensitivity of this detection technology.

The layout of the manuscript is as follows: Section 2 summarizes the Canfranc Underground Laboratory history; Section 3 focuses on those experiments using $\mathrm{NaI}(\mathrm{Tl})$ as target material applied to the search for DM; Section 4 reviews the status and prospects of ANAIS112 experiment, aiming at testing the DAMA/LIBRA result on annual modulation with more than three-sigma confidence level; and Section 5 sets the scene for the continuation of these efforts in the next years and the prospects on the mid- and long-term.

\section{The Old Canfranc Underground Facilities and the New LSC}

The Canfranc Underground Laboratory is a singular facility providing an ultra-low radioactivity environment and therefore, enabling the realization of experiments searching for very rare processes. Since the end of the 1980s, the underground facilities at the Canfranc tunnel have played a relevant role in the field of Underground Physics, hosting leading experiments in neutrinoless double beta decay $(0 v \beta \beta)$ searches [48-52], particle dark matter searches [53-72] and solar axion searches using crystalline targets [59,73]. Many other results were derived from these efforts as by-products [74-94]. For more than twenty years, the University of Zaragoza was responsible of the operation and management of the Canfranc tunnel facilities, thanks to the work of the researchers of the group in Nuclear and Astroparticle Physics, now integrated in the Center for Astroparticle and High Energy Physics (CAPA).

In 1985, researchers of the University of Zaragoza, led by Prof. Ángel Morales and Rafael Núñez-Lagos, were determined to find a convenient underground location which 
could open the door to operating their detectors in ultra-low radioactive background conditions. They followed an international trend towards this new field of research: new experiments were proposed and designed to increase the sensitivity for particle DM and $0 \nu \beta \beta$ searches, which succeeded in a few years with improvements in the sensitivity of several orders of magnitude.

The two best locations found for an underground laboratory were a salt-mine in Remolinos (near Zaragoza) and the Somport tunnel, in the railway connecting Spain and France under the Pyrenees, in Canfranc, which has not been in use since 1970. The latter location was finally chosen because of its better environmental conditions: a lower amount of dust in the air and better natural ventilation. Lab 1 (see Figure 1) was readily conditioned for immediate operation, profiting from the existence of two small halls on both sides of the tunnel ( $10 \mathrm{~m}^{2}$ each), at $780 \mathrm{~m}$ from the Spanish tunnel entrance. A few years later, Lab 2 was commissioned: a prefabricated cabin (about $15 \mathrm{~m}^{2}$ ) that could be moved onto the rails. It was first placed besides Lab 1, but afterwards, around 1991, it was joint to a second prefabricated module $\left(27 \mathrm{~m}^{2}\right)$ and moved towards higher rock overburden, at $1200 \mathrm{~m}$ from the tunnel entrance (see Figure 1).

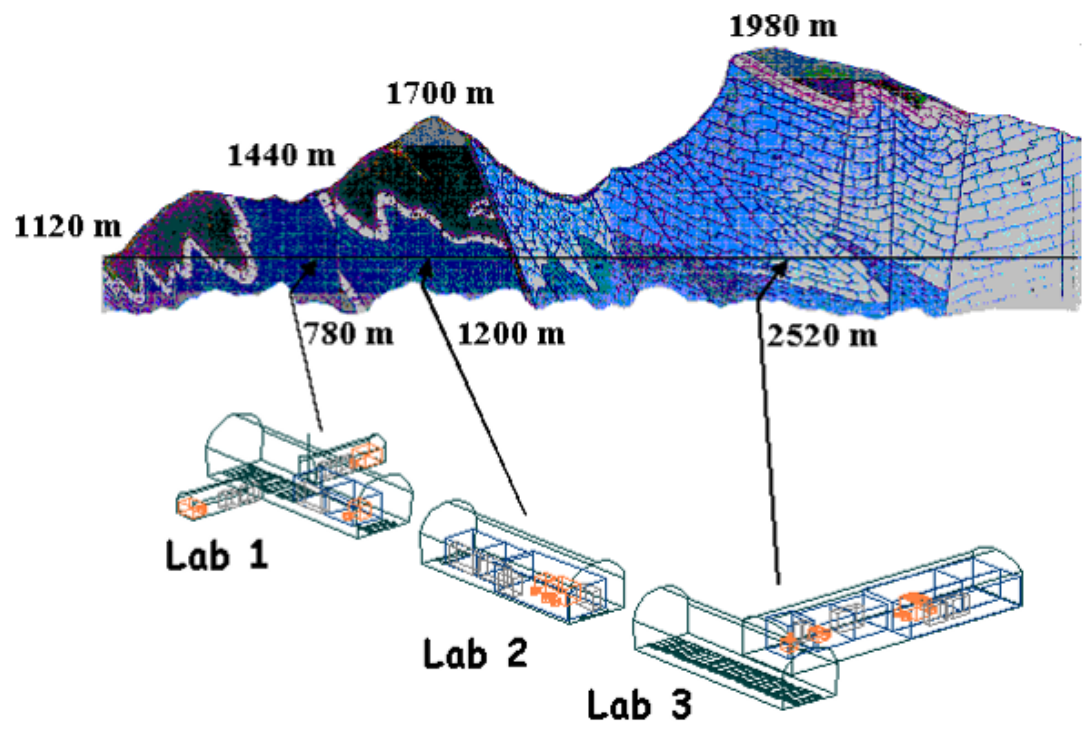

Figure 1. Scheme of the different experimental locations at the old Canfranc Underground Laboratory, in use up to 2010, except Lab 3, which is still in operation (now known as Lab2500). Distances are measured from the Spanish entrance to the Somport tunnel and rock overburden for each location is also shown.

In 1994, the excavation of a new road tunnel in the frame of a large infrastructure effort (highway from Somport to Sagunto) offered the opportunity of enlarging the underground facilities. A new experimental hall of $118 \mathrm{~m}^{2}$ was excavated and conditioned for operation (Lab 3), profiting from the maximum rock overburden the mountain profile allows (see Figure 1).

Figure 1 shows a scheme of the different experimental halls available at the Canfranc Underground Laboratory before 2010. Distances are measured from the Spanish entrance to the Somport tunnel (left side in the figure), and the rock overburden corresponding to each location is shown. Corresponding values, in meters of water equivalent (m.w.e.), are for Labs 1, 2 and 3, respectively, of 675, 1380 and 2450 m.w.e.

Starting in 2004, a new excavation to build evacuation galleries connecting the railroad tunnel with the highway tunnel allowed us to project a new enlargement of the underground facilities. This new laboratory, the only underground facility in Spain, was declared a Spanish Unique Scientific and Technical Infrastructure (ICTS) and was established as a Consortium of the Spanish Ministry of Science and Innovation, the Aragon Regional 
Government and the University of Zaragoza. In March 2006, the new facilities were inaugurated. In March 2007, the underground facilities closed because of important structural problems in the roofs of the experimental halls. Reparation works lasted until 2010, when the new facilities re-opened and access to researchers was granted again. The new Canfranc Underground Laboratory (see Figure 2) consists of more than $1000 \mathrm{~m}^{2}$ of underground space, distributed in several experimental halls, and includes a clean room class 10,000 and mechanical workshops to support the experiments [95]. Additionally, ultra-low background services (such as HPGe detectors for gamma spectroscopy or a radon abatement system) and a copper electroforming facility are offered to the users [96]. An external scientific committee is in charge of reviewing the progress of the different experiments and the evaluation of the expressions of interest. The present scientific program of LSC is focused on the study of neutrino properties (hosting experiments devoted to the investigation of the double beta decay using different techniques, CROSS [97] and NEXT [98], and collaborating in the SuperKamiokande-Gd and HyperKamiokande projects) and on the direct detection of dark matter (with the TREX-DM experiment [99] and the DArTinArDM detector [100], together with ANAIS-112). Additionally, experiments and projects related to Geophyiscs, Biology, and studies on underground muon and neutron fluxes are underway.

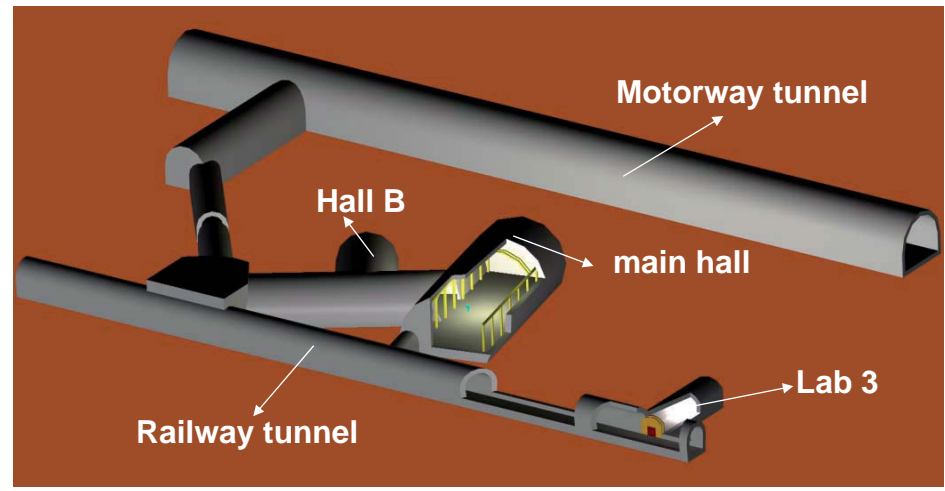

Figure 2. Scheme of the different experimental halls at the current Canfranc Underground Laboratory. Lab 3 is now called Lab2500. Main laboratory facilities (Lab2400) includes Hall A (main hall), Hall B, clean room, mechanical workshop and other services for the experiments.

\section{Direct DM Searches at the Canfranc Underground Laboratory Using NaI(T1)}

\subsection{Experiment DM32}

At the end of the 1980s, the group of Nuclear and Astroparticle Physics of the University of Zaragoza owned 14 large sodium iodide (thallium doped) detectors, $10.7 \mathrm{~kg}$ each, at the Canfranc Underground Laboratory. They were originally intended for the selection of events coincident with a central detector, in a honeycomb configuration, in a search for ${ }^{76} \mathrm{Ge} \beta \beta$ decay to excited state $2^{+}$at the Fréjus tunnel $[101,102]$. They were later used at the Canfranc Underground Laboratory, first, to discard that the Fréjus result [102] was a neutrinoless double beta decay detection [48], and after, to improve in several orders of magnitude the current limits at the time on the lifetime of $\beta^{+} \beta^{+}$and $\beta^{+}$-EC processes, by searching the decay of ${ }^{78} \mathrm{Kr}[49,103]$. The latter experiment was carried out in collaboration with the Institute of Nuclear Research (INR), Russia. At the beginning of the 1990s, a new experiment searching for DM using some of them as a target was proposed: DM32.

DM32 was a pioneer study of the annual modulation in the DM signal. It consisted of three modules $10.7 \mathrm{~kg}$ each of $\mathrm{NaI}(\mathrm{Tl})$, amounting to $32.1 \mathrm{~kg}$ of mass, and it was proposed as a proof of concept to estimate the achievable sensitivity in DM searches. The three modules were installed in a shielding consisting of $6 \mathrm{~mm}$ of Oxygen-Free High thermal Conductivity (OFHC) copper, $20 \mathrm{~cm}$ of low-activity lead, a PVC box kept at boiled-off nitrogen over-pressure, $1 \mathrm{~mm}$ of cadmium and $20 \mathrm{~cm}$ of paraffin (see Figure 3 for a detail of the inner layout of the set-up). DM32 was in operation at the Canfranc Underground Laboratory, in Lab 1 (Figure 1), under a rock overburden equivalent to $675 \mathrm{~m}$ of water, 
accumulating $4613.6 \mathrm{~kg} \times \mathrm{d}$ of data between May 1993 and December 1994. The energy threshold was at $8 \mathrm{keV}^{1}$ and the radioactive background in the low energy region was about $10 \mathrm{cpd} / \mathrm{kg} / \mathrm{keV}$ [34]. Two scenarios were considered for presenting the results of the experiment: pure spin-independent and pure spin-dependent coupling between DM particles and target nuclei, both in the standard analysis (by comparing the upper limit to the measured background to the expected signal for a given candidate) and the annual modulation analysis. The latter allowed the improvement in about two orders of magnitude of the parameter space ruled-out by the experiment $[54,55]$.

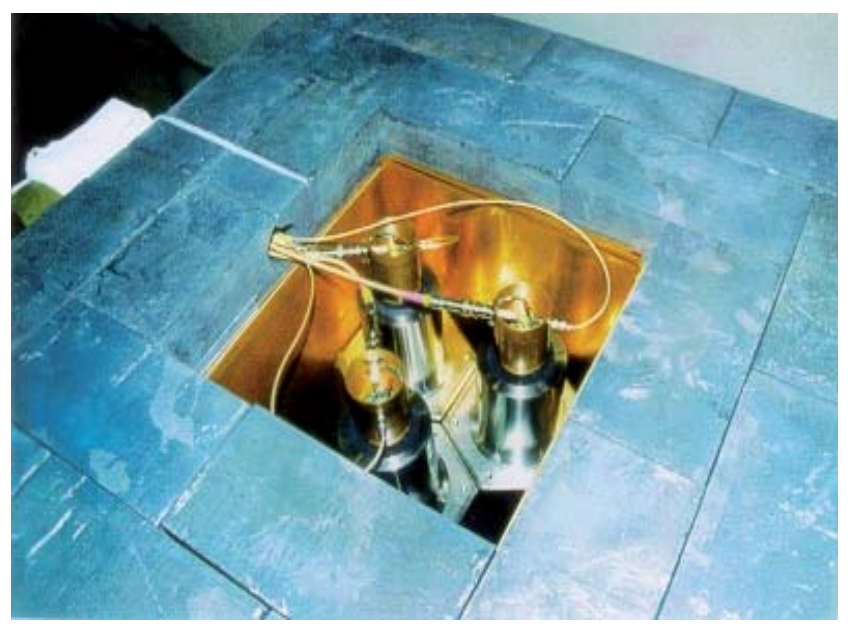

Figure 3. DM32 experiment layout: the three detectors made of $10.7 \mathrm{~kg} \mathrm{NaI}(\mathrm{Tl})$ inside the lead shielding can be seen. DM32 results can be found in $[54,55]$.

\subsection{ANAIS Prototypes}

The large expertise acquired by the group using $\mathrm{NaI}(\mathrm{Tl})$ scintillators and other techniques applied to the searches for DM pushed forward a new project-ANAIS (Annual modulation with NaI Scintillators). The rationale behind the ANAIS proposal was the positive result of annual modulation of the DAMA experiment [11,12], a few years after DM32 experiment closed operation, which has been puzzling the field for more than twenty years since then, accumulating statistical significance in its observation up to the present [13-15]. A model-independent comparison with the DAMA/LIBRA result was, from the beginning, the goal of the ANAIS project. For that goal, using the same target was mandatory, together with a different experimental approach in order to understand possible systematics. The DM32 experiment concluded that most of the observed radioactive background at low energy could be attributed to ${ }^{210} \mathrm{~Pb}$ and ${ }^{40} \mathrm{~K}$ in materials close to the crystals or in the crystals themselves. Then, the first ANAIS prototypes [35-37] tried to improve the background by reducing those contributions: replacing the PMTs and the original steel housing of the $\mathrm{NaI}(\mathrm{Tl})$ modules, made by BICRON, by more radiopure PMTs and housing. In parallel, the reduction of the energy threshold was targeted by implementing a pulse-shape discrimination procedure to distinguish PMT-origin events from NaI-scintillation events, and the design of a readout by two PMTs per module. A full program of detector upgrading, building three prototypes and carrying out measurements of the corresponding radioactive backgrounds at LSC's Lab 3 allowed us to reduce the background and threshold, but confirmed that the dominant contamination was present in the crystal, showing that an experiment using the available $150 \mathrm{~kg} \mathrm{NaI}(\mathrm{Tl})$ was not feasible. New radiopure crystal providers had to be searched for (see below). On the other hand, the effect of light guides on the light collection and a better understanding of the light propagation and optical properties of the system was achieved using those prototypes, which allowed the group to gain expertise in the design of good-performing scintillating modules. Figure 4 shows several steps of the procedure developed to couple a NaI(Tl) 
crystal to the light guides and the PMTs inside a Teflon housing, everything enclosed in a tight copper box to prevent humidity from harming the crystal (Prototype-II).
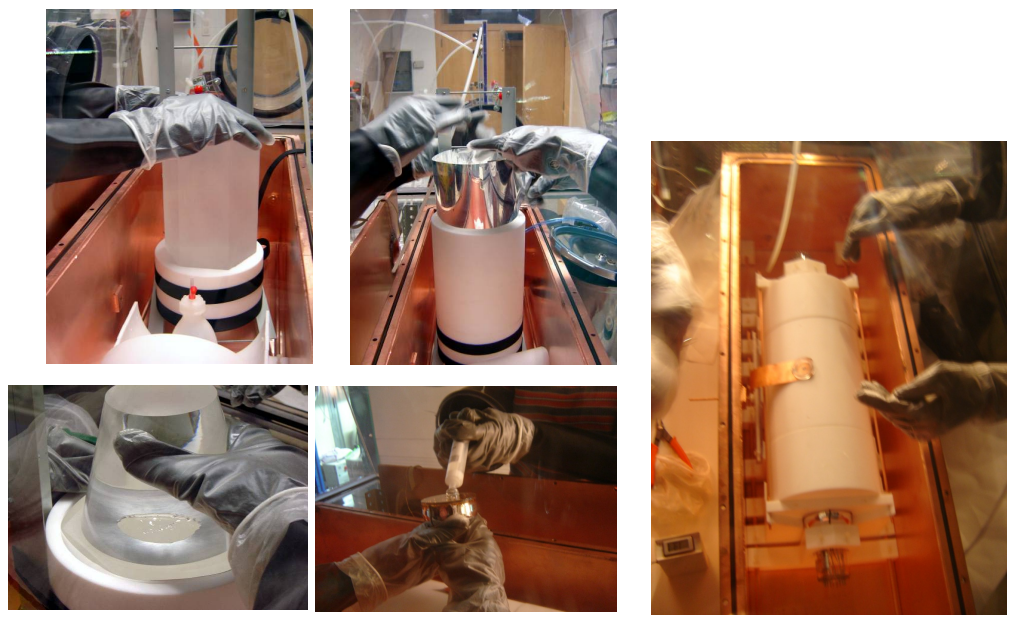

Figure 4. Process of installing Prototype-II inside a dry glove box for testing the optical performance. Later, on the Canfranc Underground Laboratory facilities, the measurements allowed to test the radioactive background of the prototype.

For instance, the scintillation of natural quartz was identified as the origin of an anomalous population of events in Prototype III, faster than $\mathrm{NaI}(\mathrm{Tl})$ bulk scintillation but slower than typical PMT-origin events ${ }^{2}$. This prototype consisted of a $10.7 \mathrm{~kg}$ BICRON crystal installed in a tight OFHC copper housing with two natural quartz optical windows allowing to couple the PMTs in a second step. Such an anomalous population was difficult to disentangle by Pulse Shape Discrimination (PSD), and imposed the careful selection of materials to design a good-performing optical system. Synthetic quartz was studied and no scintillation was found in similar conditions [89], being chosen for the optical windows in subsequent prototypes.

In parallel, as commented before, new providers of radiopure $\mathrm{NaI}(\mathrm{Tl})$ crystals were contacted, in particular, Saint Gobain, the DAMA/LIBRA crystals' provider company. A $9.6 \mathrm{~kg}$ crystal was acquired as similar as possible to those of DAMA/LIBRA, and it was used to build the Prototype-IV. This crystal was shown to be very good in terms of ${ }^{210} \mathrm{~Pb}$ bulk contamination, but not in ${ }^{40} \mathrm{~K}$. Later on, ultra-low-background $\mathrm{NaI}(\mathrm{Tl})$ crystals from Saint-Gobain, similar to those of DAMA/LIBRA, were not available, although we contacted the company on several occasions. After exploring how to develop purification protocols in collaboration with other companies, finally Alpha Spectra Inc. ${ }^{3}$ (AS), in Grand Junction, Colorado, US, was contacted. They agreed to produce NaI(Tl) following procedures proposed by our group, in particular incorporating a copper housing design with a Mylar window. Size and shape of the modules were also agreed between ANAIS team and AS. Later, this design was used to build modules for other collaborations, as COSINE. The Yale group, which was by the time involved in the DM-ice experiment [47], also acquired AS crystals but decided to change the size and mass of the detectors.

The first two AS modules arrived at LSC in 2012 and were readily characterized [38]. They showed excellent light collection but the background was higher than that of DAMA/LIBRA, mainly because of the high ${ }^{210} \mathrm{~Pb}$ contamination of the crystal itself, confirmed by the measurement of the alpha rate. Further collaboration with AS company allowed to reduce significantly this high content in ${ }^{210} \mathrm{~Pb}$ in subsequent modules, which however, remains as one of the most relevant contributions to the background budget in the region of interest (ROI), from 1 to $6 \mathrm{keV}$. Seven additional modules arrived at LSC between 2015 and 2017, completing the ANAIS-112 experiment layout, explained in detail in Section 4. 


\section{ANAIS-112 Experiment}

The ANAIS-112 experiment was the result of a time-extended R\&D effort and expertise accumulation in the operation of $\mathrm{NaI}(\mathrm{Tl})$ detectors, described in Section 3. The experiment was commissioned during the spring of 2017 and it started the data-taking phase at the hall B of the LSC on 3 August 2017.

\subsection{ANAIS-112 Experimental Setup}

A full description of ANAIS-112 experimental setup can be found in [67]. It consists of $112.5 \mathrm{~kg}$ of $\mathrm{NaI}(\mathrm{Tl})$, distributed in 9 modules, $12.5 \mathrm{~kg}$ each and built by Alpha Spectra Inc., arranged in a $3 \times 3$ configuration. Among the most relevant features of ANAIS112 modules we can mention a remarkable optical quality, which combined to using high quantum efficiency Hamamatsu photomultipliers (PMTs) results in a very high light collection, at the level of 15 photoelectrons (phe) per keV [66] in all the nine modules. Another interesting feature is a Mylar window in the middle of one of the lateral faces of the detectors, which allows to calibrate the modules with external sources of energies just few $\mathrm{keV}$ above the ROI for testing the DAMA/LIBRA result ([1-6] keV).

The ANAIS-112 experiment is installed inside a shielding consisting of an inner layer of $10 \mathrm{~cm}$ of archaeological lead and an outer layer of $20 \mathrm{~cm}$ of low activity lead. This lead shielding is encased into an anti-radon box, tightly closed and kept under overpressure with radon-free nitrogen gas. The external layer of the shielding (the neutron shielding) consists of $40 \mathrm{~cm}$ of a combination of water tanks and polyethylene bricks. An active veto made up of 16 plastic scintillators is placed between the anti-radon box and the neutron shielding, covering the top and sides of the set-up (see Figure 5) allowing to effectively tag the residual muon flux.

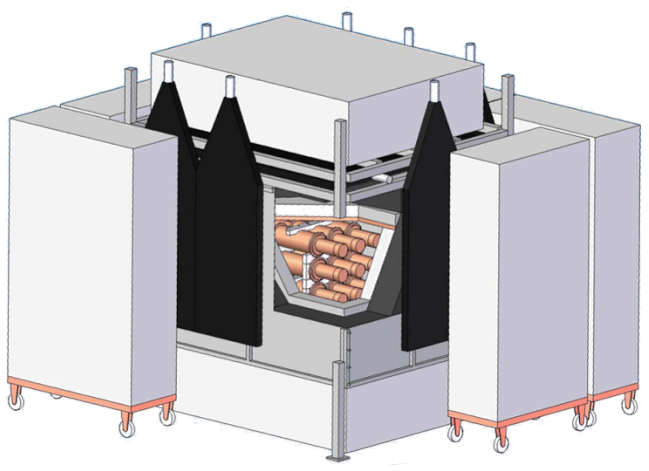

Figure 5. Sketch of the ANAIS-112 setup. It shows the NaI(Tl) modules inside a shielding made of lead, anti-radon box, active muon vetoes, and a neutron shielding made of polyethylene, and water tanks.

The signals from the two PMTs coupled to each module are digitized at $2 \mathrm{GS} / \mathrm{s}$ with high resolution. The trigger requires the coincidence of the two PMT trigger signals in a 200 ns window, while the PMT individual trigger is set at the single photo-electron level.

The stability of the experiment is guaranteed by calibrating with ${ }^{109} \mathrm{Cd}$ sources every two weeks. This allows the correction of the small gain drifts. The energy calibration is performed with the ${ }^{109} \mathrm{Cd}$ lines plus the energy depositions at very low energy (3.2 and $0.87 \mathrm{keV}$ ) associated with the decay of ${ }^{40} \mathrm{~K}$ and ${ }^{22} \mathrm{Na}$ crystal contaminations, respectively, that can be tagged by coincidences with high energy gammas. This procedure allows us to perform a reliable energy calibration of the ANAIS-112 data down to $1 \mathrm{keV}$, while the identification of the events from ${ }^{22} \mathrm{Na}$ allows us to confirm the highly efficient triggering below $1 \mathrm{keV}$. We check the stability of the calibration procedure in the ROI by calculating the residuals of the energy associated to the ${ }^{40} \mathrm{~K}$ and ${ }^{22} \mathrm{Na}$ peaks with respect to their nominal energy, gathering data every 90 days. We obtain average residuals of $0.01 \mathrm{keV}$ at the $3.2 \mathrm{keV}$ line of ${ }^{40} \mathrm{~K}$ and $-0.04 \mathrm{keV}$ at the $0.87 \mathrm{keV}$ line of ${ }^{22} \mathrm{Na}$, the latter below the ROI. The standard deviation is about $0.015 \mathrm{keV}$ for both distributions. 
The background in the ROI is dominated by non-bulk scintillation events, so we apply strong filtering protocols based on the pulse shape and light sharing among the two PMTs. The efficiency of the event selection criteria is calculated with scintillation populations $\left({ }^{109} \mathrm{Cd},{ }^{40} \mathrm{~K},{ }^{22} \mathrm{Na}\right)$ and is very close to $100 \%$ down to $2 \mathrm{keV}$, and then decreases steeply to about $15 \%$ at $1 \mathrm{keV}$, where we set the analysis threshold [67].

A careful background model of the ANAIS-112 experiment has been developed over the years, by combining inputs from different analysis techniques: HPGe screening of different materials used in the set-up and in the detector building, measurements in coincidence of the modules before ANAIS-112 commissioning, determination of alpha rate by using PSD, estimates of cosmogenic activation rates in NaI, and so forth [64,65,90,92-94]. A full description of the experiment performance after the first year and a detailed background model can be found in References $[67,68]$. Considering altogether the nine ANAIS-112 modules the average background in the ROI is $3.6 \mathrm{cpd} / \mathrm{kg} / \mathrm{keV}$ after three years of data taking [72], while DAMA/LIBRAphase2 background is below $0.80 \mathrm{cpd} / \mathrm{kg} / \mathrm{keV}$ in the [1-2] $\mathrm{keV}$ energy interval, below $0.24 \mathrm{cpd} / \mathrm{kg} / \mathrm{keV}$ in the [2-3] $\mathrm{keV}$ energy interval, and below $0.12 \mathrm{cpd} / \mathrm{kg} / \mathrm{keV}$ in the [3-4] keV energy interval [14].

\subsection{ANAIS-112 Annual Modulation Results}

The goal of ANAIS-112 experiment is the testing of the DAMA/LIBRA result on annual modulation and the experiment's design and analysis procedures have been developed having this in mind. This section summarizes the recently published annual modulation results corresponding to three years of data taking.

A blind analysis protocol has been applied: single-hit events in the ROI are kept blinded during the event selection and efficiency calculation from beginning of the data taking, but $10 \%$ of the first year's days, randomly chosen, were used for the fine-tuning of the events rejection procedures and background assessment. Up to now, we have carried out three unblindings of our data: at 1.5 years [70], at 2 years [71], and 3 years [72], which correspond to exposures of $157.55,220.69$, and $313.95 \mathrm{~kg} \times \mathrm{y}$, respectively.

We look for an annual modulation in the same energy regions as DAMA/LIBRA does: [1-6] keV and [2-6] keV with exposures of 1.13 and 2.46 ton $\times y$, respectively. We decided to group the data in 10-day bins, although later, we checked that this choice did not introduce any bias into the result. In a first approach, we worked with the total events rate, adding together the data from the nine modules, and minimize $\chi^{2}=\sum_{i}\left(n_{i}-\mu_{i}\right)^{2} / \sigma_{i}^{2}$, where $n_{i}$ is the number of events in the time bin $t_{i}$ (corrected by live time and detector efficiency), $\sigma_{i}$ is the corresponding Poisson uncertainty, accordingly corrected, and $\mu_{i}$ is the expected number of events at that time bin, that can be written as:

$$
\mu_{i}=\left[R_{0} \phi_{b k g}\left(t_{i}\right)+S_{m} \cos \left(\omega\left(t_{i}-t_{0}\right)\right)\right] M \Delta E \Delta t .
$$

Here, $R_{0}$ represents the non-modulated rate in the experiment, $\phi_{b k g}$ is the probability distribution function (PDF) in time of any non-modulated component, $S_{m}$ is the modulation amplitude, $\omega$ is fixed to $2 \pi / 365 \mathrm{~d}=0.01721 \mathrm{rad} \mathrm{d}^{-1}, t_{0}$ to $-62.2 \mathrm{~d}$ (time origin has been taken on 3 August and then the cosine maximum is on 2 June), $M$ is the total detector mass, $\Delta E$ is the energy interval width, and $\Delta t$ the time bin width. $R_{0}$ is a free parameter, while $S_{m}$ is either fixed to 0 (for the null hypothesis) or left unconstrained, positive or negative (for the modulation hypothesis).

We considered two different approaches to model the experimental background: (1) following [70,71], the background evolution is approximated to an exponential decay: $\phi_{b k g}\left(t_{i}\right)=1+f e^{-t_{i} / \tau}$, where $f$ and $\tau$ are free parameters; (2) following our Monte Carlo background model [68] the background evolution in time is computed and converted into a PDF, so $\phi_{b k g}\left(t_{i}\right)=1+f \phi_{b k g}^{M C}\left(t_{i}\right)$. Finally, (3) we considered the nine modules independently, in order to account for systematic effects that could stem from the different backgrounds and efficiencies among detectors. In this third approach, the $\chi^{2}$ summation was also 
performed over detectors and the expected number of events for every time bin $t_{i}$ and detector $d$ is written as:

$$
\mu_{i, d}=\left[R_{0, d}\left(1+f_{d} \phi_{b k g, d}^{M C}\left(t_{i}\right)\right)+S_{m} \cos \left(\omega\left(t_{i}-t_{0}\right)\right)\right] M_{d} \Delta E \Delta t,
$$

where $M_{d}$ is the mass of every module, $\phi_{b k g, d}^{M C}$ is the PDF sampled from the MC background evolution in time calculated independently for every module, and $R_{0, d}$ and $f_{d}$ are free parameters for values of $d$ between 0 and 8 .

The results of the fit for the three methods and both energy regions are shown in Figures 6-8. Results are also summarized in Table 1.
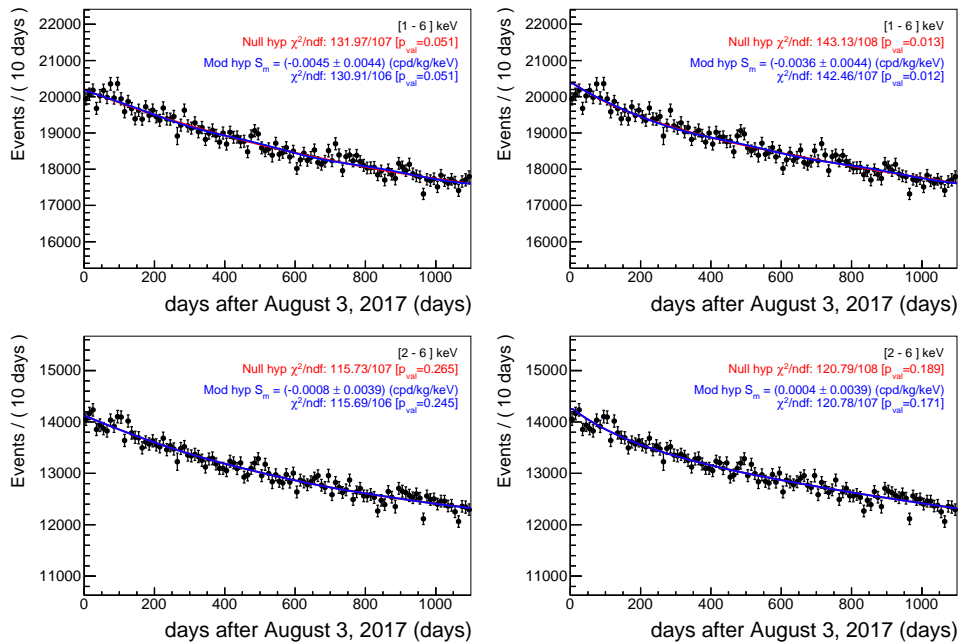

Figure 6. Upper panels: fit results for three years of data in the [1-6] keV energy region in the modulation (blue) and null hypothesis (red) when the background is described by approach (1) (left panel) or approach (2) (right panel). Lower panels: same, but for data in the [2-6] keV energy region.

Null hyp $\chi^{2} /$ ndf: $1075.81 / 972\left[p_{\text {val }}=0.011\right] \quad$ Mod hyp $\chi^{2} /$ ndf: $1075.15 / 971\left[p_{\text {val }}=0.011\right]$

$S_{m}=(-0.0034 \pm 0.0042)(\mathrm{cpd} / \mathrm{kg} / \mathrm{keV})$
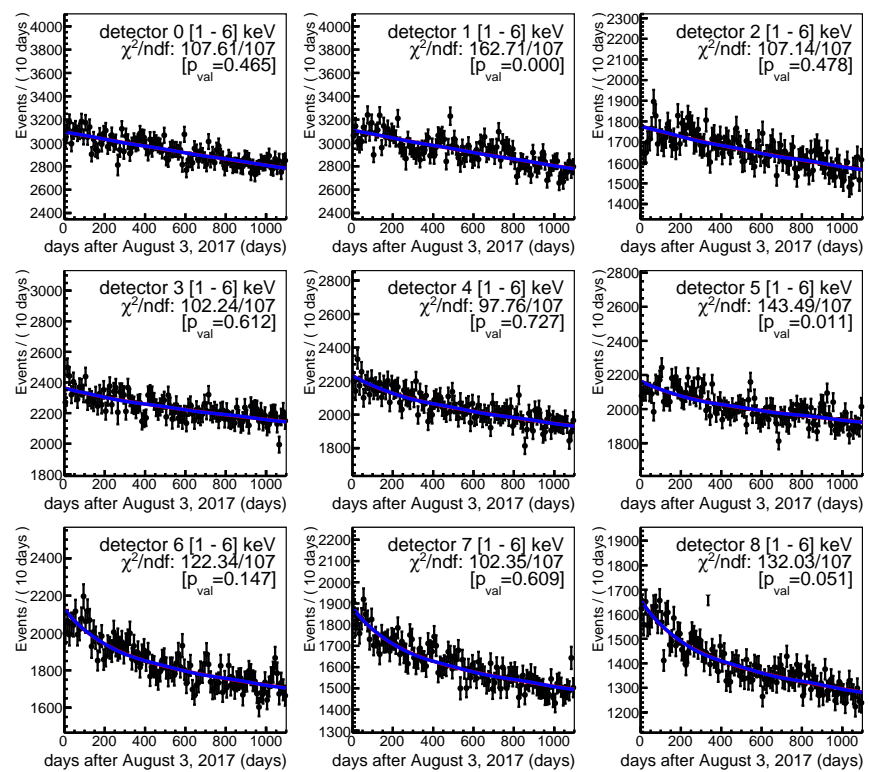

Figure 7. Fit results for three years of data in the [1-6] keV energy region in the modulation (blue) and null hypothesis (red) when the background is described by approach (3). 
Null hyp $\chi^{2 / n d f: ~} 1018.19 / 972\left[p_{\text {val }}=0.148\right] \quad$ Mod hyp $\chi^{2} /$ ndf: $1018.18 / 971\left[p_{\text {val }}=0.143\right]$

$\mathrm{S}_{\mathrm{m}}=(0.0003 \pm 0.0037)(\mathrm{cpd} / \mathrm{kg} / \mathrm{keV})$
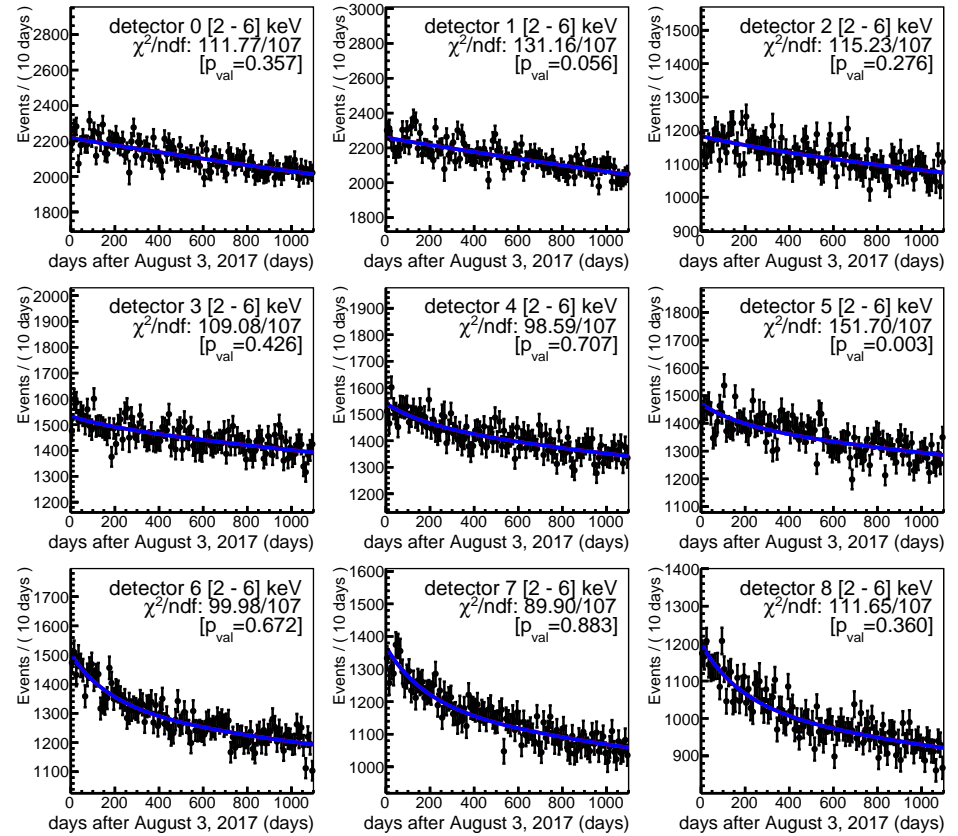

Figure 8. Fit results for three years of data in the [2-6] keV energy region in the modulation (blue) and null hypothesis (red) when the background is described by approach (3).

Table 1. Results of the fits for the null and modulation hypothesis with frequency and phase fixed in the ANAIS-112 data corresponding to three years for the three approaches followed for modelling background evolution (see text for more details).

\begin{tabular}{cccccc}
\hline Energy Region & Approach & $\chi^{2} / \mathbf{N D F}$ & $\boldsymbol{S}_{\boldsymbol{m}}$ (cpd/kg/keV) & $\boldsymbol{p}$-Value Mod & $\boldsymbol{p}$-Value Null \\
\hline$[1-6] \mathrm{keV}$ & $(1)$ & $132 / 107$ & $-0.0045 \pm 0.0044$ & 0.051 & 0.051 \\
{$[1-6] \mathrm{keV}$} & $(2)$ & $143.1 / 108$ & $-0.0036 \pm 0.0044$ & 0.012 & 0.013 \\
{$[1-6] \mathrm{keV}$} & $(3)$ & $1076 / 972$ & $-0.0034 \pm 0.0042$ & 0.011 & 0.011 \\
\hline$[2-6] \mathrm{keV}$ & $(1)$ & $115.7 / 107$ & $-0.0008 \pm 0.0039$ & 0.25 & 0.27 \\
{$[2-6] \mathrm{keV}$} & $(2)$ & $120.8 / 108$ & $0.0004 \pm 0.0039$ & 0.17 & 0.19 \\
{$[2-6] \mathrm{keV}$} & $(3)$ & $1018 / 972$ & $0.0003 \pm 0.0037$ & 0.14 & 0.15 \\
\hline
\end{tabular}

In the [2-6] keV region, the null hypothesis in the three approaches describes the data well ( $p$-values of 0.27 and 0.19 and 0.15$)$. Smaller $p$-values $(0.051,0.013$ and 0.011$)$ are obtained in [1-6] keV region. When we calculate individual $p$-values for every detector, we conclude that only detectors 1 and 5 are responsible of the low agreement with the null hypothesis in the [1-6] keV region (see Figure 7). The anomalous behavior of these two modules could be an indication of noise in the [1-2] keV energy range. The application of machine learning techniques could improve the rejection of non-bulk scintillation events contributing to ANAIS-112 background below $2 \mathrm{keV}$. We are following this new events selection strategy and the preliminary analysis points at an improvement in the p-values of these two detectors, in particular, which supports the previous hypothesis [41,104].

For the modulation hypothesis, the best fit modulation amplitudes are in all cases compatible with zero at $1 \sigma$. The standard deviation $\sigma\left(S_{m}\right)$ is the same for (1) and (2) approaches and slightly lower for the third approach, when detectors are considered independently, as expected following our a priori sensitivity analysis [69]. Therefore, this is the standard deviation chosen to quote the ANAIS-112 annual modulation final result for three years. Figure 9 shows a graphical representation of our best fits (black dots) and sensitivity (colored bands) in comparison with the DAMA/LIBRA results (blue squares). 


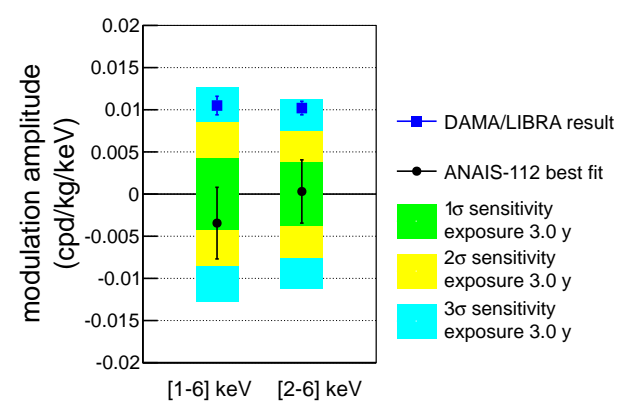

Figure 9. ANAIS-112 result on annual modulation for three years compared to DAMA/LIBRA modulation best fit. Colored bands show the ANAIS-112 estimated sensitivity for such exposure: green at $1 \sigma$, yellow at $2 \sigma$, and cyan at $3 \sigma$.

We have carried out several consistency checks (changing the number of detectors entering into the fit, considering only the first two years or the last two years, or changing the time bin size) and we conclude that we do not have any hint supporting relevant systematical uncertainties in our result. Performing a large set of Monte Carlo pseudoexperiments sampled from the background model, we have checked that the fit is not biased. When we leave the phase $t_{0}$ unconstrained, best fits are compatible with no modulation at $2 \sigma$, but a bias in the fit in the absence of modulation proportional to $\sigma\left(S_{m}\right)$, the standard deviation of the modulation amplitude, is found to be $\sqrt{\frac{\pi}{2}} \sigma\left(S_{m}\right)$. Correcting the best fits with the calculated bias, our phase-free results are compatible with no modulation in all the approaches and both energy regions at $1 \sigma$. We have also performed a frequency analysis, looking for a periodic signal at other frequencies in our data, and the conclusion is that there is no statistically significant modulation in the frequency range searched in the ANAIS-112 data [72].

\subsection{ANAIS-112 Sensitivity}

In [69] we presented a method to calculate the expected sensitivity to the annual modulation signal according to the measured background, its time evolution and the detector efficiency and live time. Following this method, we have calculated the ANAIS112 sensitivity projection as a function of the real time starting from 3 August (see Figure 10). The sensitivity is given by the ratio $S_{m}^{D A M A} / \sigma\left(S_{m}\right)$, which provides, in $\sigma$ units, the C.L. at which we can test the DAMA/LIBRA result. For three-year exposure, the ANAIS-112 result $\sigma\left(S_{m}\right)=0.0042(0.0037) \mathrm{cpd} / \mathrm{kg} / \mathrm{keV}$ for [1-6] $\mathrm{keV}$ ([2-6] keV) corresponds to $2.5 \sigma(2.7 \sigma)$ addedsensitivity.

As can be seen in Figure 10, the ANAIS-112 results for 1.5 [70], 2 [71] and 3 years [72] of data-taking fully confirm our previous analysis and this sensitivity projection.

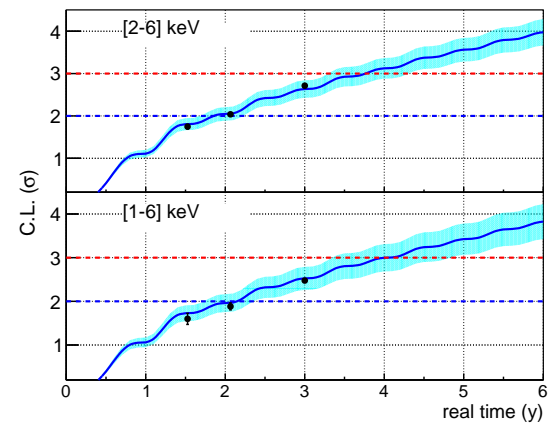

Figure 10. ANAIS-112 sensitivity to the DAMA/LIBRA signal in $\sigma$ C.L. units (see text) as a function of real time in [1-6] keV (lower panel) and [2-6] keV (upper panel) regions. The black dots are the sensitivities determined experimentally. The blue bands represent the $68 \%$ C.L. DAMA/LIBRA uncertainty. 
The statistical significance of this result increases as expected and supports our prospects of reaching a sensitivity above $3 \sigma(4 \sigma)$ in five (six) years of operation. These times can be reduced thanks to the background reduction in the [1-2] keV energy region expected from the improvement in the event selection protocols by the application of machine learning techniques. The preliminary results of this new analysis point to a background reduction of around $25 \%$ in this energy region [41].

\section{Model-Independent Testing of DAMA/LIBRA beyond $3 \sigma$ Confidence Level}

As commented before, ANAIS-112 will take data until August 2022, when five years of data will be completed and according to our estimates, statistical significance should exceed $3 \sigma$ (see Figure 10). Up to the date of writing this review, more than $95 \%$ of the time has been "live time", useful for analysis. Most of the rest, has been used for calibration of the experiment.

In order to test DAMA/LIBRA beyond $3 \sigma$ confidence level, several action lines in the frame of ANAIS-112 experiment are under consideration at present. First, we should demonstrate that ANAIS-112 is able to test DAMA/LIBRA in a fully model-independent way (see Section 5.1). Second, extending the data taking for one more year, until August 2023, will allow testing DAMA/LIBRA at the $4 \sigma$ level with the present experimental ANAIS-112 performance. However, in order to reach $5 \sigma$, a much longer period (almost 10 years in total) would be required [41].

ANAIS-112 sensitivity limitation is mostly due to the high background in the ROI, but in particular in the region from 1 to $2 \mathrm{keV}$. The ANAIS-112 background, as mentioned, is larger than that of DAMA/LIBRA mainly because of the ${ }^{210} \mathrm{~Pb}$ and ${ }^{40} \mathrm{~K}$ internal contaminations in the crystal. In addition, there is an excess of events with respect to the estimates of the background model in the region [1-2] keV [68], which could be, at least partially, attributed to non-bulk scintillation events leaking from the filtering protocols $[67,91]$. Reducing these contributions would increase the sensitivity in the [1-6] keV region. This could be achieved in different ways:

- The machine learning analysis based on Boosted Decision Trees (BDTs) under development at present, already commented in Section 4.3, could allow increasing ANAIS-112 sensitivity by reducing the contribution of this anomalous events in the [1-2] $\mathrm{keV}$ energy. This analysis could be applied to all the accumulated ANAIS-112 data;

- We are planning to modify the coupling of our PMTs to the NaI(Tl) modules, as the PMTs could be producing this anomalous population of events. ANAIS-112 sensitivity will increase if all the events excess above ANAIS-112 background model estimates disappears. If successful, this strategy would imply to start a new experimental phase, with improved background, and possibly threshold, as the main limitation for threshold reduction is the efficiency of the filtering protocols applied to remove this anomalous population;

- In parallel, we are developing R\&D activities addressing the replacement of the PMTs by SiPMs in a longer term, beyond the ANAIS-112 experiment timeline (see Section 5.2);

- In parallel, progress towards a global improvement of the background requires an upgrading in depth of the detectors, which goes beyond the ANAIS-112 experiment timeline. However, it is also the goal of complementary R\&D activities (see Section 5.3).

\subsection{Towards a Precise Knowledge of the Detector's Response}

As stressed in Section 1, a comparison between DM search results obtained with different target nuclei is affected by model-dependencies. Using the same target should allow the removal of these dependencies completely. There is, however, one caveat to this statement-a direct comparison between experiments using the same target is direct only in the case in which the response function of the detectors is fully taken into account in such a comparison of results. This issue is often disregarded, but it is very relevant in the testing of the DAMA/LIBRA result. This is because scintillation is strongly quenched for 
energy deposited by nuclear recoils with respect to the same energy deposited by electrons. Experiments are usually calibrated using gammas/electrons and, then, experimental measurements are presented in terms of electron equivalent energy $\left(\mathrm{keV}_{e e}\right)$ and experiments can be directly compared only if energy is deposited by this channel. In the case when DM particles produce nuclear recoils, quenching factors (QF) should be well known to re-calibrate the energy depositions scale.

This is not the case for NaI scintillators. Measurements of QF in the energy region relevant for DM searches, and in particular for the comparison with DAMA/LIBRA in case the signal is attributed to nuclear recoils, do not agree, and in fact are affected by strong discrepancies (QF dependent on the energy versus constant values, for instance) [105-110]. We are collaborating with Duke and Yale researchers to measure more accurately the QF of several crystals in the same conditions in order to determine whether specific crystal properties traduce in a different QF. Preliminary results have been presented recently, but further work in this line is foreseen [111].

In addition, calibrations with neutrons onsite could play a relevant role in DM searches. In the last period of operation of ANAIS-112 we will develop a more complete calibration program using neutron sources.

\subsection{RED Activities to Improve NaI(Tl) Detector Technology: Replacing PMTs by SiPMs}

$\mathrm{NaI}(\mathrm{Tl})$ detectors are at present state-of-the-art radiation detectors with many applications in very different fields, from environmental monitoring to medical diagnosis. However, their performance for application in rare event searches has progressed slowly. Radiopurity of DAMA/LIBRA crystals has not yet been improved by any other group, for instance, although SABRE, PICOLON and COSINE-200 collaborations are progressing steadily in this direction, growing crystals independently and developing their own protocols [112-114]. As mentioned above, one of the main limitations at present of the application of $\mathrm{NaI}(\mathrm{Tl})$ in rare event searches is the light sensor, the PMT. Replacing the PMTs with SiPMs would bring many relevant advantages: lower mass is associated with a lower background contribution from possible radioactive contaminants, quantum efficiency is higher in the wavelength of interest for the readout of $\mathrm{NaI}(\mathrm{Tl})$ scintillation, and spurious events originating in the PMTs could be avoided. On the other hand, SiPMs are affected by a much larger dark rate at room temperature, hindering their application for low-energy processes. Very good results have been obtained by cooling the SiPMs in terms of general performance, but in particular in the dark rate. Because of that, efforts to develop a new light readout of $\mathrm{NaI}(\mathrm{Tl})$ crystals using SiPMs have started at the University of Zaragoza. The goal is to operate the $\mathrm{NaI}(\mathrm{Tl})$ or intrinsic $\mathrm{NaI}$ crystal coupled to the SiPMs at very low temperatures-around $100 \mathrm{~K}$-trying to profit from the low dark rate of the SiPM and the light yield increase that could be achieved (at least for the intrinsic NaI) in that temperature range. Working at that temperature is a challenge, but it opens very interesting possibilities - a liquid Argon/Xenon bath could be used simultaneously as active veto shielding and thermal bath, allowing for an additional background rejection strategy. The Canfranc Underground Laboratory disposes of a large tank designed to operate liquid Ar, in the frame of the ArDM [115] and DarkSide [100] projects, that could be used for the testing of a prototype.

\subsection{RED Activities to Improve NaI(Tl) Detector Technology: Growing NaI(Tl) Crystals Underground}

Crystal growing for application in rare event searches requires the use of a radiopure powder as input and an extreme ultra-low background environment. Powder purification procedures are usually applied before the growing. Then, it is particularly important to control crucible radiopurity and possible presence of radon in the atmosphere. In addition, re-crystallization and zone-refining procedures can strongly contribute to more radiopure final crystals [116]. 
However, cosmogenically activated isotopes are being continuously produced while powder and crystals are at the surface, and more significantly if the growing place is at high altitude over sea level, as happens in Grand Junction. It is very interesting to have the possibility of growing crystals underground, shielded from cosmic rays. In the case of $\mathrm{NaI}(\mathrm{Tl})$, we have identified that a relevant part of our background in the ROI is associated with tritium, produced cosmogenically [93], but many other isotopes are produced [92]. Although most of them are short-lived, they explain the exponentially decaying background of ANAIS-112 (see Figures 6-8).

We are collaborating on a project to grow radiopure $\mathrm{NaI}(\mathrm{Tl})$ crystals underground, at the facilities of the Canfranc Underground Laboratory.

\section{Summary and Outlook}

Many years of expertise in the operation of $\mathrm{NaI}(\mathrm{Tl})$ scintillation detectors at the Canfranc Underground Laboratory support the results of the ANAIS-112 experiment on the model-independent testing of the DAMA/LIBRA observation of an annual modulation with all the expected features corresponding to a DM signal. Five years of data taking will be completed in 2022, allowing for the first time this testing at a $3 \sigma$ confidence level. No hint of the presence of modulation in ANAIS-112 (three-year exposure analysis) can be reported. The present status of the ANAIS-112 experiment and prospects to continue this research have been presented in this manuscript.

Author Contributions: This manuscript reviews more than thirty years of research in different experiments carried out at the Canfranc Underground Laboratory using $\mathrm{NaI}(\mathrm{Tl})$ scintillation detectors. The list of authors includes those who passed away too soon: Á.M., J.M. and J.Á.V. We are deeply in debt with their pioneering work and vision. All the other authors have read and agreed to the published version of the manuscript. Á.M., J.M. and J.Á.V. contributed to get funds for the experiments and the underground facilities' operation. Á.M., J.M., J.Á.V., E.G., A.O.d.S. and M.L.S. contributed to the design of the experiments; J.M., J.A., A.O.d.S., M.L.S., A.S., S.C., M.M., C.P., C.C., M.Á.O. and P.V. contributed to the setting-up and commissioning of the experiments; J.M., J.A., D.C., C.C., M.M., M.Á.O., T.P., C.P., Y.O., A.O.d.S., M.L.S. and S.C. performed calibration and maintenance of the experiments; J.M., M.M., C.P. and M.Á.O. developed the DAQ software tools; I.C., C.C., M.Á.O., C.P., M.M. and M.L.S. developed analysis tools; E.G., I.C., C.C., M.M., C.P., J.P. and M.L.S. analyzed the data; S.C., C.C. and P.V. developed background simulation codes; E.G., A.O.d.S. and J.P. performed radiopurity measurements; I.C., E.G., M.M. and J.P. contributed to sensitivity estimates. All authors have read and agreed to the published version of the manuscript.

Funding: This research was funded by MCIN/AEI/10.13039/501100011033 under grant PID2019104374GB-I00; by MINECO-FEDER under grants FPA2017-83133-P, and FPA2014-55986-P; by MICINNFEDER under grants FPA2011-23749, and FPA2008-03228; by MEC under grants FPA2007-63777, and FPA2004-00974; by CICYT under grants FPA2001-2437, AEN99-1033, AEN98-0627, AEN95-9747, AEN93-0363, and AEN92-0084; by CONSOLIDER-Ingenio 2010 Programme under grants MultiDark CSD2009-00064 and CPAN CSD2007-00042; by Gobierno de Aragón and the European Social Fund; and by the LSC consortium.

Data Availability Statement: ANAIS-112 data are available upon request. After the scheduled five-year data taking, data will be released.

Acknowledgments: All the work here reviewed has been possible thanks to a large collective effort to support activities at the Canfranc Underground Laboratory for more than thirty years. Many researchers have contributed then, more or less directly, to this work and are worth to be acknowledged. In particular, authors would like to highlight the contributions from Rafael NúnezLagos, Carlos Sáenz, Eva Cerezo, Igor García Irastorza, Stefano Scopel, Héctor Gómez, Asunción Rodríguez, Lidia Torres, Carlos Ginestra, and Diana Carolina Herrera. In addition, authors would like to acknowledge the use of Servicio General de Apoyo a la Investigación-SAI, Universidad de Zaragoza, and technical support from LSC staff.

Conflicts of Interest: The authors declare no conflict of interest. The founding sponsors had no role in the design of the study, collection, analysis or interpretation of data, writing of the manuscript or decision to publish. 


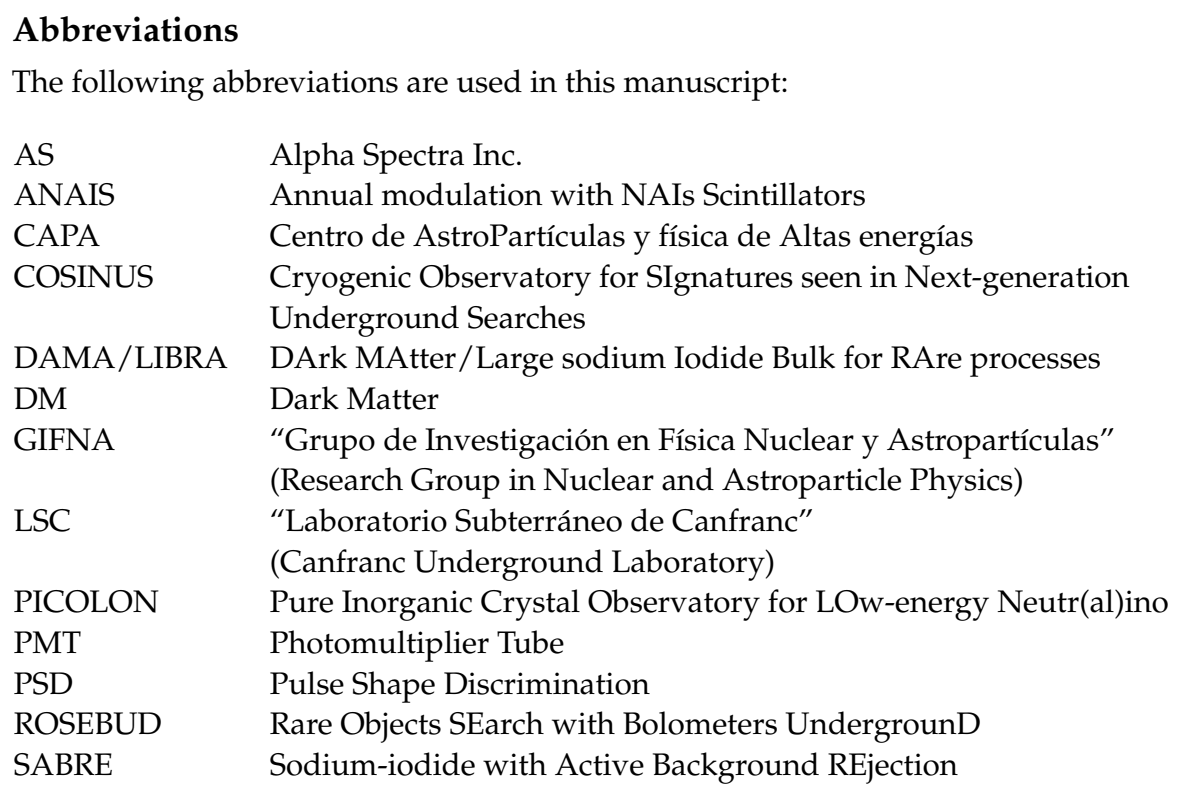

\section{Notes}

1 Unless otherwise stated, throughout this paper, all the energies shown will correspond to electron equivalent energies. This issue is further commented in Section 5.1.

2 These are mainly associated with dark events and Cherenkov emission in the PMT glass being the pulse shape dominated by the Single Electron Response of the PMT.

3 https://alphaspectra.com/ (accessed date: 17 January 2022).

\section{References}

1. Zyla, P.A. et al. [Particle Data Group]. The Review of Particle Physics. Prog. Theor. Exp. Phys. 2020, 2020, 083C01. [CrossRef]

2. Bertone, G.; Hooper, D. History of dark matter. Rev. Mod. Phys. 2018, 90, 045002. [CrossRef]

3. Buchmueller, O.; Doglioni, C.; Wang, L.T. Search for dark matter at colliders. Nat. Phys. 2017, 13, 217. [CrossRef]

4. Conrad, J.; Reiner, O. Indirect dark matter searches in gamma and cosmic rays. Nat. Phys. 2017, 13, 224. [CrossRef]

5. Liu, J.; Chen, X.; Ji, X. Current status of direct dark matter detection experiments. Nat. Phys. 2017, 13, 212. [CrossRef]

6. Schumann, M. Direct Detection of WIMP Dark Matter: Concepts and Status. J. Phys. G Nucl. Part. Phys. 2019, $46,1103003$. [CrossRef]

7. Billard, J.; Boulay, M.; Cebrián, S.; Covi, L.; Fiorillo, G.; Green, A.; Kopp, J.; Majorovits, B.; Palladino, K.; Petricca, F.; et al. Direct Detection of Dark Matter-APPEC Committee Report. arXiv 2021, arXiv:2104.07634.

8. Aprile, E.; et al. [XENON Collaboration]. Excess electronic recoil events in XENON1T. Phys. Rev. D 2020, 102, 072004 [CrossRef]

9. Choi, G.; Suzuki, M.; Yanagida, T.T. XENON1T anomaly and its implication for decaying warm dark matter. Phys. Lett. B 2020, 811, 135976. [CrossRef]

10. Freese, K.; Frieman, J.; Gould, A. Signal modulation in cold-dark-matter detection. Phys. Rev. D 1988, 37, 3388. [CrossRef]

11. Bernabei, R. et al. [DAMA Collaboration]. Performance of the approximately 100kg NaI(Tl) set-up of the DAMA experiment at Gran Sasso. Riv. Nuovo C. 1999, 111, 545.

12. Bernabei, R. et al. [DAMA Collaboration]. Search for WIMP annual modulation signature: Results from DAMA/NaI-3 and DAMA/NaI-4 and the global combined analysis. Phys. Lett. B 2000, 480, 23. [CrossRef]

13. Bernabei, R. et al. [DAMA Collaboration]. First results from DAMA/LIBRA and the combined results with DAMA/NaI. Eur. Phys. J. C 2008, 56, 333. [CrossRef]

14. Bernabei, R.; Belli, P.; Bussolotti, A.; Cappella, F.; Caracciolo, V.; Cerulli, R.; Dai, C.J.; d'Angelo, A.; Di Marco, A.; Ferrari, N.; et al. The DAMA project: Achievements, implications and perspectives. Prog. Part. Nucl. Phys. 2020, 114, 103810. [CrossRef]

15. Bernabei, R.; Belli, P.; Bussolotti, A.; Cappella, F.; Caracciolo, V.; Cerulli, R.; Dai, C.J.; d'Angelo, A.; Di Marco, A.; He, H.L.; et al. First model independent results from DAMA/LIBRA-phase2. Nucl. Phys. At. Energy 2018, 19, 307. [CrossRef]

16. Bernabei, R.; Belli, P.; Caracciolo, V.; Cerulli, R.; Merlo, V.; Cappella, F.; d'Angelo, A.; Incichitti, A.; Dai, C.J.; Ma, X.H.; et al. The dark matter: DAMA/LIBRA and its perspectives. arXiv 2021, arXiv:2110.04734.

17. Cui, X. et al. [PandaX-II Collaboration]. Dark Matter Results From 54-Ton-Day Exposure of PandaX-II Experiment. Phys. Rev. Lett. 2017, 119, 181302. [CrossRef]

18. Meng, Y. et al. [PandaX-4T Collaboration]. Dark Matter Search Results from the PandaX-4T Commissioning Run. Phys. Rev. Lett. 2021, 127, 261802. [CrossRef] 
19. Aprile, E. et al. [XENON Collaboration]. Light Dark Matter Search with Ionization Signals in XENON1T. Phys. Rev. Lett. 2019, 123, 251801. [CrossRef]

20. Aprile, E. et al. [XENON Collaboration]. Dark Matter Search Results from a One Ton-Year Exposure of XENON1T. Phys. Rev. Lett. 2018, 121, 111302. [CrossRef]

21. Akerib, D.S. et al. [LUX Collaboration]. Results from a search for dark matter in the complete LUX exposure. Phys. Rev. Lett. 2017, 118, 021303. [CrossRef] [PubMed]

22. Ajaj, R. et al. [DEAP Collaboration]. Search for dark matter with a 231-day exposure of liquid argon using DEAP-3600 at SNOLAB Phys. Rev. D 2019, 100, 022004. [CrossRef]

23. Agnes, P. et al. [DarkSide Collaboration]. DarkSide-50 532-day Dark Matter Search with Low-Radioactivity Argon. Phys. Rev. D 2018, 98, 102006. [CrossRef]

24. Agnes, P. et al. [DarkSide Collaboration]. Low-Mass Dark Matter Search with the DarkSide-50 Experiment. Phys. Rev. Lett. 2018, 121, 081307. [CrossRef]

25. Agnese, R. et al. [SuperCDMS Collaboration]. Search for Low-Mass Weakly Interacting Massive Particles with SuperCDMS. Phys. Rev. Lett. 2014, 112, 241302. [CrossRef]

26. Agnese, R. et al. [SuperCDMS Collaboration]. Search for Low-Mass Dark Matter with CDMSlite Using a Profile Likelihood Fit Phys. Rev. D 2019, 99, 062001. [CrossRef]

27. Armengaud, E. et al. [EDELWEISS Collaboration]. Searching for low-mass dark matter particles with a massive Ge bolometer operated above-ground. Phys. Rev. D 2019, 99, 082003. [CrossRef]

28. Abdelhameed, A.H. et al. [CRESST Collaboration]. First results from the CRESST-III low-mass dark matter program. Phys. Rev. D 2019, 100, 102002. [CrossRef]

29. Adhikari, G. et al. [COSINE-100 Collaboration]. Strong constraints from COSINE-100 on the DAMA dark matter results using the same sodium iodide target. Sci. Adv. 2021, 7, abk2699. [CrossRef]

30. Aprile, E. et al. [XENON Collaboration]. Search for Electronic Recoil Event Rate Modulation with 4 Years of XENON100 Data Phys. Rev. Lett. 2017, 118, 101101. [CrossRef]

31. Kobayashi, M. et al. [XMASS Collaboration]. Search for sub-GeV dark matter by annual modulation using XMASS-I detector. Phys. Lett. B 2019, 795, 308. [CrossRef]

32. Akerib, D.S. et al. [LUX Collaboration]. Search for annual and diurnal rate modulations in the LUX experiment. Phys. Rev. D 2018, 98, 062005. [CrossRef]

33. Yang, L.T. et al. [CDEX Collaboration]. Search for Light Weakly-Interacting-Massive-Particle Dark Matter by Annual Modulation Analysis with a Point-Contact Germanium Detector at the China Jinping Underground Laboratory. Phys. Rev. Lett. 2019, 123, 221301. [CrossRef] [PubMed]

34. Sarsa, M.L. Experimento Para la Detección Directa de Materia Oscura Galáctica fría con Detectores de Centelleo Mediante la búsqueda de Señales Distintivas. Ph.D. Thesis, Universidad de Zaragoza, Zaragoza, Spain, December 1995.

35. Cebrián, S. Estudio del Fondo Radiactivo en Experimentos Subterráneos de búsqueda de Sucesos Poco Probables: CUORE (Cryogenic Underground Observatory for Rare Events) y ANAIS (Annual Modulation with NaIs). Ph.D. Thesis, Universidad de Zaragoza, Zaragoza, Spain, January 2002.

36. Pobes, C. Optimización de la Respuesta de un Sistema Multidetector de Cristales de Centelleo Para un Experimento de Materia Oscura (ANAIS). Ph.D. Thesis, Universidad de Zaragoza, Zaragoza, Spain, May 2006.

37. Martínez, M. Diseño de un Prototipo para un Experimento de Detección Directa de Materia Oscura Mediante Modulación Anual con Centelleadores de Ioduro de Sodio. Ph.D. Thesis, Universidad de Zaragoza, Zaragoza, Spain, July 2006.

38. Cuesta, C. ANAIS-0: Feasibility Study for a $250 \mathrm{~kg} \mathrm{NaI(Tl)}$ Dark Matter Search Experiment at the Canfranc Underground Laboratory. Ph.D. Thesis, Universidad de Zaragoza, Zaragoza, Spain, May 2013.

39. Oliván, M.A. Design, Scale-up and Characterization of the Data Acquisition System for the ANAIS Dark Matter Experiment. Ph.D. Thesis, Universidad de Zaragoza, Zaragoza, Spain, December 2015.

40. Villar, P. Background Evaluation of the ANAIS Dark Matter Experiment in Different Configurations: Towards a Final Design. Ph.D. Thesis, Universidad de Zaragoza, Zaragoza, Spain, December 2016.

41. Coarasa, I. ANAIS-112. Searching for the Annual Modulation of Dark Matter with a $112.5 \mathrm{~kg} \mathrm{NaI(Tl)} \mathrm{Detector} \mathrm{at} \mathrm{the} \mathrm{Canfranc}$ Underground Laboratory. Ph.D. Thesis, Universidad de Zaragoza, Zaragoza, Spain, December 2021.

42. Adhikari, G. et al. [COSINE-100 Collaboration]. Search for a Dark Matter-Induced Annual Modulation Signal in NaI(Tl) with COSINE-100 Experiment. Phys. Rev. Lett. 2019, 123, 031302. [CrossRef]

43. Adhikari, G. et al. [COSINE-100 Collaboration]. Three-year annual modulation search with COSINE-100. arXiv 2021, arXiv:2111.08863

44. Antonello, M. et al. [SABRE Collaboration]. The SABRE project and the SABRE Proof-of-Principle. Eur. Phys. J. C 2019, 79, 36.

45. Fushimi, K.I. Low Background Measurement by Means of NaI(Tl) Scintillator: Improvement of Sensitivity for Cosmic Dark Matter. Radioisotopes 2018, 67, 101. [CrossRef]

46. Angloher, G.; Carniti, P.; Cassina, L.; Gironi, L.; Gotti, C.; Gütlein, A.; Hauff, D.; Maino, M.; Nagorny, S.S.; Pagnanini, L.; et al. The COSINUS project - perspectives of a NaI scintillating calorimeter for dark matter search. Eur. Phys. J. C 2016, 76, 441. [CrossRef]

47. Barbosa de Souza, E. et al. [DM_Ice Collaboration]. First search for a dark matter annual modulation signal with $\mathrm{NaI}(\mathrm{Tl})$ in the Southern Hemisphere by DM-Ice17. Phys. Rev. D 2017, 95, 032006. [CrossRef] 
48. Morales, A.; Morales, J.; Núñez-Lagos, R.; Puimedón, J.; Villar, J.A.; Larrea, A.; García, E.; Sáenz, C. Results of a search of the neutrinoless decay of ${ }^{76} \mathrm{Ge}$ to the first excited state of ${ }^{76} \mathrm{Se}$ in the Canfranc tunnel. Il Nuovo C. A 1991, 104, 1581. [CrossRef]

49. Sáenz, C.; Cerezo, E.; García, E.; Morales, A.; Morales, J.; Núñez-Lagos, R.; Ortiz de Solórzano, A.; Puimedón, J.; Salinas, A.; Sarsa, M.L.; et al. Results of a search for double positron decay electron-positron conversion of ${ }^{78}$ Kr. Phys. Rev. C 1994, $50,1170$. [CrossRef] [PubMed]

50. Aalseth, C.E. et al. [IGEX Collaboration]. Neutrinoless double beta decay of ${ }^{76} \mathrm{Ge}$ : First results from the International Germanium Experiment (IGEX) with isotopically enriched detectors. Phys. Rev. C 1999, 59, 2108. [CrossRef]

51. Aalseth, C.E. et al. [IGEX Collaboration]. The IGEX ${ }^{76} \mathrm{Ge}$ neutrinoless double-beta decay experiment: Prospects for next generation experiments. Phys. Rev. D 2002, 65, 092007. [CrossRef]

52. Cebrián, S. Double beta decay experiments at Canfranc Underground Laboratory. Prog. Part. Nucl. Phys. 2020, $114,103807$. [CrossRef]

53. García, E.; Morales, A.; Morales, J.; Sarsa, M.L.; Ortiz de Solórzano, A.; Cerezo, E.; Núñez-Lagos, R.; Puimedón, J.; Sáenz, C.; Salinas, A.; et al. Results of a Dark Matter Search with a Germanium detector in the Canfranc Tunnel. Phys. Rev. D 1995, $51,1458$. [CrossRef]

54. Sarsa, M.L.; Morales, A.; Morales, J.; García, E.; Ortiz de Solórzano, A.; Puimedón, J.; Sáenz, C.; Salinas, A.; Villar, J.A. Searching for annual modulation of WIMPs with NaI scintillators. Phys. Lett. B 1996, 386, 458. [CrossRef]

55. Sarsa, M.L.; Morales, A.; Morales, J.; García, E.; Ortiz de Solórzano, A.; Puimedón, J.; Sáenz, C.; Salinas, A.; Villar, J.A. Results of a search for annual modulation of WIMP signals. Phys. Rev. D 1997, 56, 1856. [CrossRef]

56. Cebrián, S.; Coron, N.; Dambier, G.; García, E.; González, D.; Irastorza, I.G.; Leblanc, J.; de Marcillac, P.; Morales, A.; Morales, J.; et al. Performances and prospects of the ROSEBUD dark matter search experiment. Astrop. Phys. 1999, 10, 361. [CrossRef]

57. Morales, A. et al. [IGEX Collaboration]. New constraints on WIMPs from the Canfranc IGEX Dark Matter Search. Phys. Lett. $B$ 2000, 489, 268. [CrossRef]

58. Cebrián, S.; Coron, N.; Dambier, G.; García, E.; González, D.; Irastorza, I.G.; Leblanc, J.; de Marcillac, P.; Morales, A.; Morales, J.; et al. First results of the ROSEBUD Dark Matter experiment. Astrop. Phys. 2001, 15, 79. [CrossRef]

59. Morales, A. et al. [COSME Collaboration]. Particle dark matter and solar axion searches with a small germanium detector at the Canfranc Underground Laboratory. Astrop. Phys. 2002, 16, 325. [CrossRef]

60. Morales, A.; Aalseth, C.E.; Avignone, F.T., III; Brodzinski, R.L.; Cebrián, S.; García, E.; Irastorza, I.G.; Kirpichnikov, I.V.; Klimenko, A.A.; Miley, H.S.; et al. Improved constraints on WIMPs from the International Germanium Experiment IGEX. Phys. Lett. B 2001, 532, 8. [CrossRef]

61. Cebrián, S.; Amaré, J.; Carmona, J.M.; García, E.; Irastorza, I.G.; Luzón, G.; Morales, A.; Morales, J.; Ortiz de Solórzano, A.; Puimedón, J.; et al. Status and preliminary results of the ANAIS experiment at Canfranc. Nucl. Phys. B (Proc. Suppl.) 2002, 110, 94. [CrossRef]

62. Cebrián, S.; Coron, N.; Dambier, G.; de Marcillac, P.; García, E.; Irastorza, I.G.; Leblanc, J.; Morales, A.; Morales, J.; Ortiz de Solórzano, A.; et al. First underground light versus heat discrimination for dark matter search. Phys. Lett. B 2003, 563, 48. [CrossRef]

63. Cebrián, S.; Coron, N.; Dambier, G.; García, E.; Irastorza, I.G.; Leblanc, J.; de Marcillac, P.; Morales, A.; Morales, J.; Ortiz de Solórzano, A.; et al. Bolometric WIMP search at Canfranc with different absorbers. Astrop. Phys. 2004, 21, 23. [CrossRef]

64. Cebrián, S.; Cuesta, C.; Amaré, J.; Borjabad, S.; Fortuño, D.; García, E.; Ginestra, C.; Gómez, H.; Martínez, M.; Oliván, M.A.; et al. Background model for a $\mathrm{NaI}(\mathrm{Tl})$ detector devoted to dark matter searches. Astrop. Phys. 2012, 37, 60. [CrossRef]

65. Amaré, J.; Cebrián, S.; Cuesta, C.; García, E.; Martínez, M.; Oliván, M.A.; Ortigoza, Y.; Ortiz de Solórzano, A.; Puimedón, J.; Sarsa, M.L.; et al. Assessment of backgrounds of the ANAIS experiment for dark matter direct detection. Eur. Phys. J. C 2016, 76, 429. [CrossRef]

66. Oliván, M.A.; Amaré, J.; Cebrián, S.; Cuesta, C.; García, E.; Martínez, M.; Ortigoza, Y.; Ortiz de Solórzano, A.; Pobes, C.; Puimedón, J.; et al. Light yield determination in large sodium iodide detectors applied in the search for dark matter. Astrop. Phys. 2017, 93, 86-95. [CrossRef]

67. Amaré, J.; Cebrián, S.; Coarasa, I.; Cuesta, C.; García, E.; Martínez, M.; Oliván, M.A.; Ortigoza, Y.; Ortiz de Solórzano, A.; Puimedón, J.; et al. Performance of ANAIS-112 experiment after the first year of data taking. Eur. Phys. J. C 2019, 79, 228. [CrossRef]

68. Amaré, J.; Cebrián, S.; Coarasa, I.; Cuesta, C.; García, E.; Martínez, M.; Oliván, M.A.; Ortigoza, Y.; Ortiz de Solórzano, A.; Puimedón, J.; et al. Analysis of backgrounds for the ANAIS-112 dark matter experiment. Eur. Phys. J. C 2019, 79, 412. [CrossRef]

69. Coarasa, I.; Cebrián, S.; Coarasa, I.; Cuesta, C.; García, E.; Martínez, M.; Oliván, M.A.; Ortigoza, Y.; Ortiz de Solórzano, A.; Puimedón, J.; et al. ANAIS-112 sensitivity in the search for dark matter annual modulation. Eur. Phys. J. C 2019, 79, 233. [CrossRef]

70. Amaré, J.; Cebrián, S.; Coarasa, I.; Cuesta, C.; García, E.; Martínez, M.; Oliván, M.A.; Ortigoza, Y.; Ortiz de Solórzano, A.; Puimedón, J.; et al. First results on dark matter annual modulation from ANAIS-112 experiment. Phys. Rev. Lett. 2019, 123, 031301. [CrossRef] [PubMed]

71. Amaré, J.; Cebrián, S.; Cintas, D.; Coarasa, I.; García, E.; Martínez, M.; Oliván, M.A.; Ortigoza, Y.; Ortiz de Solórzano, A.; Puimedón, J.; et al. ANAIS-112 status: Two years results on annual modulation. J. Phys. Conf. Ser. 2020, 1468, 012014. [CrossRef] 
72. Amaré, J.; Cebrián, S.; Cintas, D.; Coarasa, I.; García, E.; Martínez, M.; Oliván, M.A.; Ortigoza, Y.; Ortiz de Solórzano, A.; Puimedón, J.; et al. Annual modulation results from three-year exposure of ANAIS-112. Phys. Rev. D 2021, 103, 102005. [CrossRef]

73. Cebrián, S.; García, E.; González, D.; Irastorza, I.G.; Morales, A.; Morales, J.; Ortiz de Solórzano, A.; Puimedón, J.; Salinas, A.; Sarsa, M.L.; et al. Prospects of solar axion searches with crystal detectors. Astrop. Phys. 1999, 10, 397. [CrossRef]

74. Aharonov, Y.; Avignone, F.T.; Brodzinski, R.L.; Collar, J.I.; García, E.; Miley, H.S.; Morales, A.; Morales, J.; Nussinov, S.; Ortiz de Solórzano, A.; et al. New experimental limits for the electron stability. Phys. Lett. B 1995, 353, 168. [CrossRef]

75. Aharonov, Y.; Avignone, F.T.; Brodzinski, R.L.; Collar, J.I.; García, E.; Miley, H.S.; Morales, A.; Morales, J.; Nussinov, S.; Ortiz de Solórzano, A.; et al. New laboratory bounds on the stability of the electron. Phys. Rev. D 1995, 52, 3785. [CrossRef]

76. Abriola, D.; Avignone, F.T.; Brodzinski, R.L.; Collar, J.I.; Di Gregorio, D.E.; Farach, H.A.; García, E.; Gattone, A.O.; Hasenbalg, F.; Huck, H.; et al. Searching for Cold Dark Matter in the Southern Hemisphere. The experiment at Sierra Grande. Astrop. Phys. 1996, 6, 63. [CrossRef]

77. Abriola, D.; Avignone, F.T.; Brodzinski, R.L.; Collar, J.I.; Di Gregorio, D.E.; Farach, H.A.; García, E.; Gattone, A.O.; Guérard, C.K.; Hasenbalg, F.; et al. Search for an annual modulation of dark matter signals with a germanium spectrometer at the Sierra Grande Laboratory. Astrop. Phys. 1999, 10, 133. [CrossRef]

78. Cebrián, S.; García, E.; González, D.; Irastorza, I.G.; Morales, A.; Morales, J.; Ortiz de Solórzano, A.; Peruzzi, A.; Puimedón, J.; Sarsa, M.L.; et al. Sensitivity plots for WIMP direct detection using the annual modulation signature. Astrop. Phys. 2001, 14, 339. [CrossRef]

79. Cebrián, S.; Coron, N.; Dambier, G.; de Marcillac, P.; García, E.; Irastorza, I.G.; Leblanc, J.; Morales, A.; Morales, J.; Ortiz de Solórzano, A.; et al. Improved limits for natural $\alpha$ radioactivity of tungsten with a $\mathrm{CaWO}_{4}$ scintillating bolometer. Phys. Lett. B 2003, 556, 14. [CrossRef]

80. Carmona, J.M.; Cebrián, S.; García, E.; Irastorza, I.G.; Luzón, G.; Morales, A.; Morales, J.; Ortiz de Solórzano, A.; Puimedón, J.; Sarsa, M.L.; et al. Neutron background at the Canfranc Underground Laboratory and its contribution to the IGEX-DM dark matter experiment. Astrop. Phys. 2004, 21, 523. [CrossRef]

81. Amaré, J.; Beltrán, B.; Cebrián, S.; García, E.; Gómez, H.; Irastorza, I.G.; Luzón, G.; Martínez, M.; Morales, J.; Ortiz de Solórzano, A.; et al. Light yield of undoped sapphire at low temperature under particle excitation. Appl. Phys. Lett. 2005, 87, 264102. [CrossRef]

82. Coron, N.; García, E.; Gironnet, J.; Leblanc, J.; de Marcillac, P.; Martínez, M.; Ortigoza, Y.; Pobes, C.; Puimedón, J.; Redon, T.; et al Thermal relative efficiency factor for recoiling ${ }^{206} \mathrm{~Pb}$ nuclei in a sapphire bolometer. Phys. Lett. B 2008, 659, 113. [CrossRef]

83. Coron, N.; García, E.; Gironnet, J.; Leblanc, J.; de Marcillac, P.; Martínez, M.; Ortigoza, Y.; Ortiz de Solórzano, A.; Pobes, C.; Puimedón, J.; et al. A BGO scintillating bolometer as dark matter detector prototype. Opt. Mater. 2009, 31, 1393. [CrossRef]

84. Ortigoza, Y.; Coron, N.; Cuesta, C.; García, E.; Ginestra, C.; Gironnet, J.; de Marcillac, P.; Martínez, M.; Pobes, C.; Puimedón, J.; et al. Energy partition in sapphire and BGO scintillation bolometers. Astrop. Phys. 2011, 34, 603. [CrossRef]

85. Coron, N.; Cuesta, C.; García, E.; Ginestra, C.; Gironnet, J.; de Marcillac, P.; Martínez, M.; Ortigoza, Y.; Pobes, C.; Puimedón, J.; et al. Measurement of the L/K electron-capture ratio of the ${ }^{207} \mathrm{Bi}$ decay to the $1633 \mathrm{keV}$ level of ${ }^{207} \mathrm{~Pb}$ with a BGO scintillating bolometer. Eur. Phys. J. A 2012, 48, 89. [CrossRef]

86. Cerdeño, D.G.; Cuesta, C.; Fornasa, M.; García, E.; Ginestra, C.; Huh, J.H.; Martínez, M.; Ortigoza, Y.; Peiró, M.; Puimedón, J.; et al Complementarity of dark matter direct detection: The role of bolometric targets. J. Cosmol. Astropart. Phys. 2013, 7, 28. [CrossRef]

87. Cuesta, C.; Oliván, M.A.; Amaré, J.; Cebrián, S.; García, E.; Ginestra, C.; Martínez, M.; Ortigoza, Y.; Ortiz de Solórzano, A.; Pobes, C.; et al. Slow scintillation time constants in NaI(Tl) for different interacting particles. Opt. Mat. 2013, 36, 316. [CrossRef]

88. Coron, N.; Cuesta, C.; García, E.; Ginestra, C.; Girard, T.A.; de Marcillac, P.; Martínez, M.; Ortigoza, Y.; Ortiz de Solórzano, A.; Pobes, C.; et al. Study of Parylene-coated NaI(Tl) at low temperatures for bolometric applications. Astrop. Phys. 2013, 47, 31. [CrossRef]

89. Amaré, J.; Borjabad, S.; Cebrián, S.; Cuesta, C.; Fortuño, D.; García, E.; Ginestra, C.; Gómez, H.; Herrera, D.C.; Martínez, M.; et al. Study of scintillation in natural and synthetic quartz and methacrylate. Opt. Mat. 2014, 36, 1408. [CrossRef]

90. Cuesta, C.; Amaré, J.; Cebrián, S.; García, E.; Ginestra, C.; Martínez, M.; Oliván, M.A.; Ortigoza, Y.; Ortiz de Solórzano, A.; Pobes, C.; et al. Analysis of the ${ }^{40} \mathrm{~K}$ contamination in $\mathrm{NaI}(\mathrm{Tl})$ crystals from different providers in the frame of the ANAIS project. Int. J. Mod. Phys. A 2014, 29, 1443010/01. [CrossRef]

91. Cuesta, C.; Amaré, J.; Cebrián, S.; García, E.; Ginestra, C.; Martínez, M.; Oliván, M.A.; Ortigoza, Y.; Ortiz de Solórzano, A.; Pobes, C.; et al. Bulk NaI(Tl) scintillation low energy events selection with the ANAIS-0 module. Eur. Phys. J. C 2014, 74, 3150. [CrossRef]

92. Amaré, J.; Cebrián, S.; Cuesta, C.; García, E.; Ginestra, C.; Martínez, M.; Oliván, M.A.; Ortigoza, Y.; Ortiz de Solórzano, A.; Pobes, C.; et al. Cosmogenic radionuclide production in NaI(Tl) crystals. J. Cosmol. Astropart. Phys. 2015, 2, 46. [CrossRef]

93. Amaré, J.; Castel, J.; Cebrián, S.; Coarasa, I.; Cuesta, C.; Dafni, T.; Galán, J.; García, E.; Garza, J.G.; Iguaz, F.J.; et al. Cosmogenic production of tritium in dark matter detectors. Astrop. Phys. 2018, 97, 96. [CrossRef]

94. Villar, P.; Amaré, J.; Cebrián, S.; Coarasa, I.; García, E.; Martínez, M.; Oliván, M.A.; Ortigoza, Y.; Ortiz de Solórzano, A.; Puimedón, J.; et al. Study of the cosmogenic activation in NaI(Tl) crystal within the ANAIS experiment. Int. J. Mod. Phys. A 2018, $33,1843006$. [CrossRef]

95. Ianni, A. The Canfranc Underground Laboratory: A multidisciplinary underground facility. J. Phys. Conf. Ser. 2016, 675, 012002. [CrossRef] 
96. Bandac, I.; Borjabad, S.; Ianni, A.; Núñez-Lagos, R.; Pérez, C.; Rodríguez, S.; Villar, J.A. Ultra-low background and environmental measurements at Laboratorio Subterráneo de Canfranc (LSC). Appl. Radiat. Isot. 2017, 126, 127. [CrossRef]

97. Bandac, I.; et al. [CROSS Collaboration]. The 0v2 $\beta$-decay CROSS experiment: Preliminary results and prospects. J. High Energy Phys. 2020, 01, 018.

98. Adams, C. et al. [NEXT Collaboration]. Sensitivity of a tonne-scale NEXT detector for neutrinoless double-beta decay searches. J. High Energy Phys. 2021, 8, 164.

99. Castel, J.; Cebrián, S.; Coarasa, I.; Dafni, T.; Galán, J.; Iguaz, F.J.; Irastorza, I.G.; Luzón, G.; Mirallas, H.; Ortiz de Solórzano, A.; et al. Background assessment for the TREX dark matter experiment. Eur. Phys. J. C 2019, 79, 782. [CrossRef]

100. Aalseth, C.E. et al. [DarkSide-20k Collaboration]. Design and construction of a new detector to measure ultra-low radioactiveisotope contamination of argon. J. Instrum. 2020, 15, P02024. [CrossRef]

101. Morales, A.; Morales, J.; Núñez-Lagos, R.; Puimedón, J.; Villar, J.A.; Larrea, A. Results of a search on the neutrinoless double beta decay of ${ }^{76} \mathrm{Ge}$ to the excited states of ${ }^{76}$ Se. Nuovo C. A 1988, 100, 525. [CrossRef]

102. Busto, J.; Dassie, D.; Helene, O.; Hubert, P.; Larrieu, P.; Leccia, F.; Mennrath, P.; Aleonard, M.M.; Chevallier, J. The $0^{+}->2^{+}$ neutrinoless double beta decay of ${ }^{76} \mathrm{Ge}$ : Results of the Fréjus experiment. Nucl. Phys. A 1990, 513, 291. [CrossRef]

103. Sáenz, C. Investigación experimental sobre la conversión electrón-positrón y la doble desintegración beta en ${ }^{78} \mathrm{Kr}$. Ph.D. Thesis, Universidad de Zaragoza, Zaragoza, Spain, July 1994.

104. Coarasa, I.; Apilluelo, J.; Amaré, J.; Cebrián, S.; Cintas, D.; García, E.; Martínez, M.; Oliván, M.A.; Ortigoza, Y.; Ortiz de Solórzano, A.; et al. Machine-learning techniques applied to three-year exposure of ANAIS-112. arXiv 2021, arXiv:2110.10649.

105. Spooner, N.; Davies, G.J.; Davies, J.D.; Pyle, G.J.; Bucknell, T.D.; Squier, G.T.A.; Lewin, J.D.; Smith, P.F. The scintillation efficiency of sodium and iodine recoils in a $\mathrm{NaI}(\mathrm{Tl})$ detector for dark matter searches. Phys. Lett. 1994, 321, 156. [CrossRef]

106. Chagani, H.; Majewski, P.; Daw, E.J.; Kudryavtsev, V.A.; Spooner, N.J.C. Measurement of the quenching factors of Na recoils in $\mathrm{NaI}(\mathrm{Tl})$. J. Instrum. 2008, 3, 06003. [CrossRef]

107. Collar, J.I. Quenching and channeling of nuclear recoils in NaI(Tl): Implications for dark-matter searches. Phys. Rev. C 2013, 88 , 035806. [CrossRef]

108. Xu, J.; Shields, E.; Calaprice, F.; Westerdale, S.; Froborg, F.; Suerfu, B.; Alexander, T.; Aprahamian, A.; Back, H.O.; Casarella, C.; et al. Scintillation efficiency measurement of $\mathrm{Na}$ recoils in $\mathrm{NaI}(\mathrm{Tl})$ below the DAMA/LIBRA energy threshold. Phys. Rev. C 2015, 92, 015807. [CrossRef]

109. Joo, H.W.; Park, H.S.; Kim, J.H.; Lee, J.H.; Kim, S.K.; Kim, Y.D.; Lee, H.S.; Kim, S.H. Quenching factor measurement for NaI(Tl) scintillation crystal. Astrop. Phys. 2019, 108, 50. [CrossRef]

110. Bignell, L.J.; Mahmood, I.; Nuti, F.; Lane, G.J.; Akber, A.; Barberio, E.; Baroncelli, T.; Coombes, B.J.; Dix, W.; Dowie, J.T.H.; et al. Quenching factor measurements of sodium nuclear recoils in NaI:Tl determined by spectrum fitting. J. Instrum. 2021, 16, 07034. [CrossRef]

111. Cintas, D.; An, P.; Awe, C.; Barbeau, P.S.; Barbosa de Souza, E.; Hedges, S.; Jo, J.H.; Martínez, M.; Maruyama, R.; Li, L.; et al. Quenching factor consistency across several NaI(Tl) crystals. arXiv 2021, arXiv:2111.09590.

112. Suerfu, B.; Wada, M.; Peloso, W.; Souza, M.; Calaprice, F.; Tower, J.; Ciampi, G. Growth of ultra-high purity NaI(Tl) crystals for dark matter searches. Phys. Rev. Res. 2020, 2, 013223. [CrossRef]

113. Fushimi, K.; Kanemitsu, Y.; Hirata, S.; Chernyak, D.; Hazama, R.; Ikeda, H.; Imagawa, K.; Ishiura, H.; Ito, H.; Kisimoto, T.; et al. Development of highly radiopure $\mathrm{NaI}(\mathrm{Tl})$ scintillator for PICOLON dark matter search project. Prog. Theor. Exp. Phys. 2021, 2021, 043F01. [CrossRef]

114. Park, B.J. et al. [COSINE Collaboration]. Development of ultra-pure NaI(Tl) detector for COSINE-200 experiment. Eur. Phys. J. C 2020, 80, 814. [CrossRef]

115. Calvo, J. et al. [ArDM Collaboration]. Commissioning of the ArDM experiment at the Canfranc Underground Laboratory: First steps towards a tonne-scale liquid argon time projection chamber for Dark Matter Searches. J. Cosmol. Astropart. Phys. 2017, 3, 003.

116. Suerfu, B.; Calaprice, F.; Souza, M. Zone Refining of Ultrahigh-Purity Sodium Iodide for Low-Background Detectors. Phys. Rev. Appl. 2021, 16, 014060. [CrossRef] 